\title{
Sex without crossing over in the yeast Saccharomycodes ludwigii
}

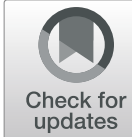

Ioannis A. Papaioannou ${ }^{1}$, Fabien Dutreux ${ }^{2}$, France A. Peltier ${ }^{1}$, Hiromi Maekawa ${ }^{1,3}$, Nicolas Delhomme ${ }^{4}$, Amit Bardhan ${ }^{1}$, Anne Friedrich ${ }^{2}$, Joseph Schacherer ${ }^{2,5}$ and Michael Knop ${ }^{1,6^{*}}$ (i)

* Correspondence: m.knop@zmbh.
uni-heidelberg.de
${ }^{1}$ Center for Molecular Biology of
Heidelberg University (ZMBH),
Heidelberg, Germany
${ }^{6}$ German Cancer Research Center
(DKFZ), DKFZ-ZMBH Alliance,
Heidelberg, Germany
Full list of author information is
available at the end of the article

* Correspondence: m.knop@zmbh. uni-heidelberg.de Heidelberg Unive Heidelberg, Germany ${ }^{6}$ German Cancer Research Cente (DKFZ), DKFZ-ZMBH Alliance, Full list of author information is available at the end of the article

\begin{abstract}
Background: Intermixing of genomes through meiotic reassortment and recombination of homologous chromosomes is a unifying theme of sexual reproduction in eukaryotic organisms and is considered crucial for their adaptive evolution. Previous studies of the budding yeast species Saccharomycodes ludwigii suggested that meiotic crossing over might be absent from its sexual life cycle, which is predominated by fertilization within the meiotic tetrad.

Results: We demonstrate that recombination is extremely suppressed during meiosis in $\mathrm{Sd}$. ludwigii. DNA double-strand break formation by the conserved transesterase Spo11, processing and repair involving interhomolog interactions are required for normal meiosis but do not lead to crossing over. Although the species has retained an intact meiotic gene repertoire, genetic and population analyses suggest the exceptionally rare occurrence of meiotic crossovers in its genome. A strong AT bias of spontaneous mutations and the absence of recombination are likely responsible for its unusually low genomic GC level.

Conclusions: Sd. ludwigii has followed a unique evolutionary trajectory that possibly derives fitness benefits from the combination of frequent mating between products of the same meiotic event with the extreme suppression of meiotic recombination. This life style ensures preservation of heterozygosity throughout its genome and may enable the species to adapt to its environment and survive with only minimal levels of rare meiotic recombination. We propose $S d$. ludwigii as an excellent natural forum for the study of genome evolution and recombination rates.
\end{abstract}

Keywords: Achiasmate meiosis, Automixis, Crossing over, Intratetrad mating, Meiotic recombination, Linkage disequilibrium, Mutation accumulation, Mutation rate, Saccharomycodes ludwigii

\section{Background}

Sex is the prevailing reproductive mode throughout the eukaryotic tree of life [1]. At its core lies a periodic ploidy cycling, accomplished through meiosis, during which haploid gametes are produced, and mating, which ensures the restoration of the original ploidy level. Meiosis, thought to have arisen early in the eukaryotic evolution, is a ubiquitous attribute of sexual life cycles [2]. During the first meiotic division (meiosis

(c) The Author(s). 2021 Open Access This article is licensed under a Creative Commons Attribution 4.0 International License, which permits use, sharing, adaptation, distribution and reproduction in any medium or format, as long as you give appropriate credit to the original author(s) and the source, provide a link to the Creative Commons licence, and indicate if changes were made. The images or other third party material in this article are included in the article's Creative Commons licence, unless indicated otherwise in a credit line to the material. If material is not included in the article's Creative Commons licence and your intended use is not permitted by statutory regulation or exceeds the permitted use, you will need to obtain permission directly from the copyright holder. To view a copy of this licence, visit http://creativecommons.org/licenses/by/4.0/. The Creative Commons Public Domain Dedication waiver (http://creativecommons.org/publicdomain/zero/1.0/) applies to the data made available in this article, unless otherwise stated in a credit line to the data. 
I), parental chromosomes are recombined and separated, while in the second division sister chromatids segregate (meiosis II). Reassortment and recombination of homologous chromosomes in meiosis I lead to novel genetic constellations in the offspring. These function as substrates for natural selection, which can promote advantageous and purge deleterious genetic combinations [3, 4]. However, the considerable biological costs of sex render its widespread occurrence paradoxical, and the questions of its evolutionary origin and functions are outstanding enigmas in biology [5-7].

Saccharomyces cerevisiae (baker's yeast) has been used as a premier model organism to gain major insights into the mechanisms of meiosis [8, 9]. Following pairing of homologous chromosomes, meiotic recombination is initiated in prophase I with the formation of DNA double-strand breaks (DSBs) by the topoisomerase-like transesterase Spo11 [10]. Several pathways mediate the repair of these DSBs and pathway choice is regulated by a multitude of meiosis-specific factors that act in concert with the DNA repair machinery. One possibility involves the use of the homologous chromosome as repair template, which can lead to the generation of chimeric DNA molecules. This process may involve either reciprocal exchange of the chromosomal regions that flank the DSB site (crossover, CO) or gene conversion without reciprocal exchange (noncrossover, NCO) [8, 9]. Crossing over establishes chiasmata (Fig. 1d), which ensure the physical interconnection of bivalents that is important for the faithful segregation of homologous chromosomes in meiosis I [13]. In many, but not all, organisms, this is facilitated by the synaptonemal complex (SC), which mediates stable synapsis [9].

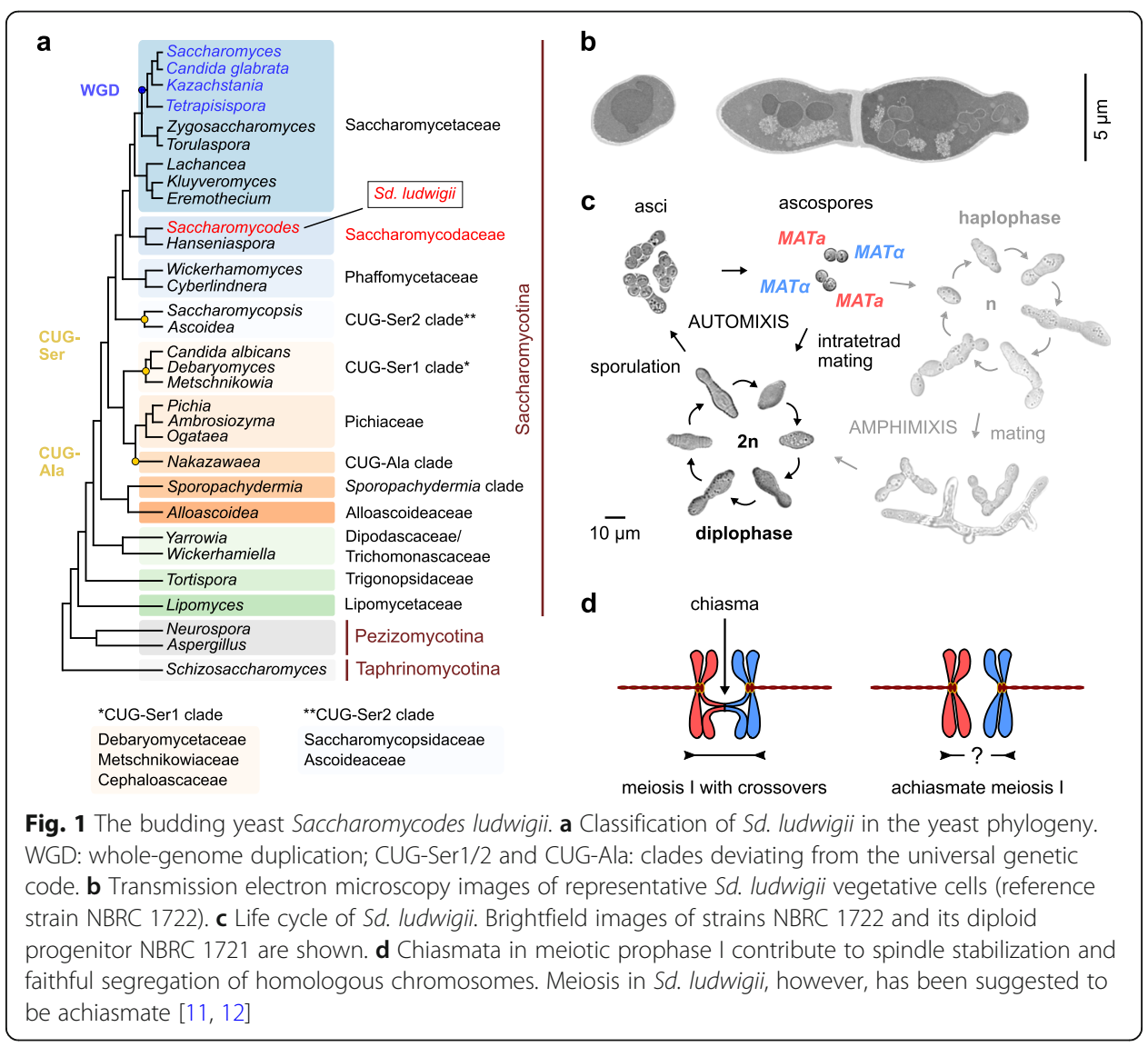


The extent of genetic exchange in meiosis and thus generation of genomic diversity depend on the frequency and distribution of COs along chromosomes. Homeostatic regulation controls these parameters in many organisms [14, 15], with different outcomes between species. These range from one obligatory $\mathrm{CO}$ per chromosome in Caenorhabditis elegans [16] to 10 or more in Schizosaccharomyces pombe [17], and up to 15 in the longest chromosomes of S. cerevisiae [18]. Furthermore, generation of diversity is influenced by the mating behavior (breeding), which can involve gametes of variable genetic relatedness. Outbreeding involves mating between unrelated gametes, ensuring high genetic diversity in the offspring. On the other hand, inbreeding (selffertilization) refers to mating between gametes that originate from the same individual or clonal line. Inbreeding generally reduces heterozygosity, which can compromise the adaptive potential of populations [19].

Mating of gametes from the same meiotic event represents a particular type of inbreeding referred to as intratetrad mating or automixis [20], the most frequent breeding strategy in S. cerevisiae [21-23]. If automixis brings together chromosomes that had been separated during meiosis I (non-sister components), it is described as "central fusion" or "first division restitution". This has important genetic consequences, since it maintains parental heterozygosity around the centromeres, reducing the risk of deleterious alleles being exposed due to homozygotization [24-26]. The degree to which parental heterozygosity is restituted upon intratetrad mating correlates inversely with the frequency of COs during meiosis. In the absence of crossing over, parental genomes would be fully reconstituted and their heterozygosity maintained. Evolutionary models suggested that high frequency of deleterious mutations could promote automixis in the absence of meiotic recombination [27].

Chromosome segregation in meiosis I without crossing over (achiasmate meiosis) has been observed in one of the two sexes $[6,28]$ or individual chromosomes $[29,30]$ of a few organisms. Unusually low overall recombination rates have also been reported in some species [31, 32], but these data should be interpreted with caution since experimental limitations such as sampling bias and insufficient marker coverage may have hindered the identification of COs in many of these cases. In addition, most of these studies could not exclude the possibility of crossing over near chromosome ends [31].

The budding yeast species Saccharomycodes ludwigii (Fig. 1a, b) preferentially undergoes intratetrad mating (Fig. 1c), ensured by strong interspore bridges that efficiently keep spores together in pairs of opposite mating types within meiotic tetrads [33, 34]. This organism was used in the early days of yeast genetics for the pioneering description of heterothallism by Øjvind Winge at the Carlsberg laboratory [34, 35]. During his studies, Winge also observed in this species an unusual segregation pattern of two cell morphology markers [34]. The subsequent interpretation by Lindegren was that the two genes were not linked and that "each is so close to the spindle attachment that segregation invariably occurs at the Meiosis I without crossing over" [36]. Later work by the Oshima laboratory surprisingly revealed that the same behavior, which would be consistent with centromere linkage, was displayed by any combination of more than 20 genetic markers tested. These findings led to the conclusion that this "may be due to the absence of crossing over in Sd. ludwigii" [11, 12].

Here we explored the extent and types of meiotic interactions between homologous chromosomes in Sd. ludwigii. For this, we performed whole-genome sequencing and 
high-contiguity genome assembly of wild-type strains, which enabled a high-resolution DNA variant segregation analysis of meiosis. We combined bioinformatic analyses of the meiotic gene repertoire with a functional study of key meiotic components in order to derive a better understanding of the meiotic mechanisms in this species. We also searched for signs of recombination on an evolutionary scale between divergent strains of Sd. ludwigii in comparison to other species with different levels of meiotic recombination. To gain insights into the relative contributions of mutational pressure and recombination to genome evolution, we determined the genome-wide mutation rate and bias using a mutation accumulation experiment. Our results provide insights into the unique sexual lifestyle of the yeast species $S d$. ludwigii, and they propose this organism as a particularly suitable model system for the study of the evolution of recombination rates and their impact on genome evolution.

\section{Results}

\section{The Sd. ludwigii genome}

To investigate meiotic recombination in Sd. ludwigii, we first used long-read PacBio sequencing to generate a high-contiguity de novo genome assembly of our reference haploid strain NBRC 1722 (Additional file 1: Table S1 [11, 37-39]). The 12.5-Mb assembly consists of 7 chromosomal scaffolds, in concordance with PFGE karyotyping [12, 40] (Fig. 2a) and previous genetic mapping [11, 12]. Gene synteny and DNA motif analyses (Additional file 2: Fig. S1a) enabled the identification of putative point centromeres on all chromosomes. The extremities of all scaffolds share subtelomere-related repetitive sequences and genes, while telomeric DNA repeats were also detected in 9 cases (Additional file 2: Fig. S1b). A single mating-type locus (MATalpha) was identified in the centromeric vicinity of chrE (Fig. 2a), in congruence with the known heterothallic nature of the species $[34,35]$.

The Sd. ludwigii genome shares many features with Saccharomycetaceae members that diverged before the whole-genome duplication event (Fig. 1a), including genome size, number of chromosomes, point centromeres, overall gene synteny, number of genes (5031 ORFs), and frequency of introns (3.3\%). Remarkably, Sd. ludwigii chromosomes exhibit GC levels that are unusually low for a yeast species (30.9\% on average; Fig. 2a), as shown by a comparison to 100 yeast and other fungal genomes (Additional file 2: Fig. S2a; Additional file 3: Table S2). The comparative analysis further revealed exceptionally high coverage in $S d$. ludwigii by AT-rich low-complexity regions and simple sequence repeats (microsatellites), as well as enrichment in transposable elements (Additional file 2: Fig. S2b-e).

Meiotic crossing over could be hindered in chromosomal regions of Sd. ludwigii that do not align during meiotic prophase I due to major sequence variation or structural differences of the homologous chromosomes. To investigate whether high genomic dissimilarity could be responsible for the scarcity of COs observed in previous analyses $[11,12]$ that used our reference strain as one of the parents, we also sequenced and assembled de novo the genome of the second parent used in those experiments (NBRC 1723; Additional file 1: Table S1). The two parental genomes are highly collinear (Fig. 2b) and similar at the DNA sequence level (99.6\% identity on average), apart from the terminal region of chrA and the longest part of chrF, which exhibit lower degrees 


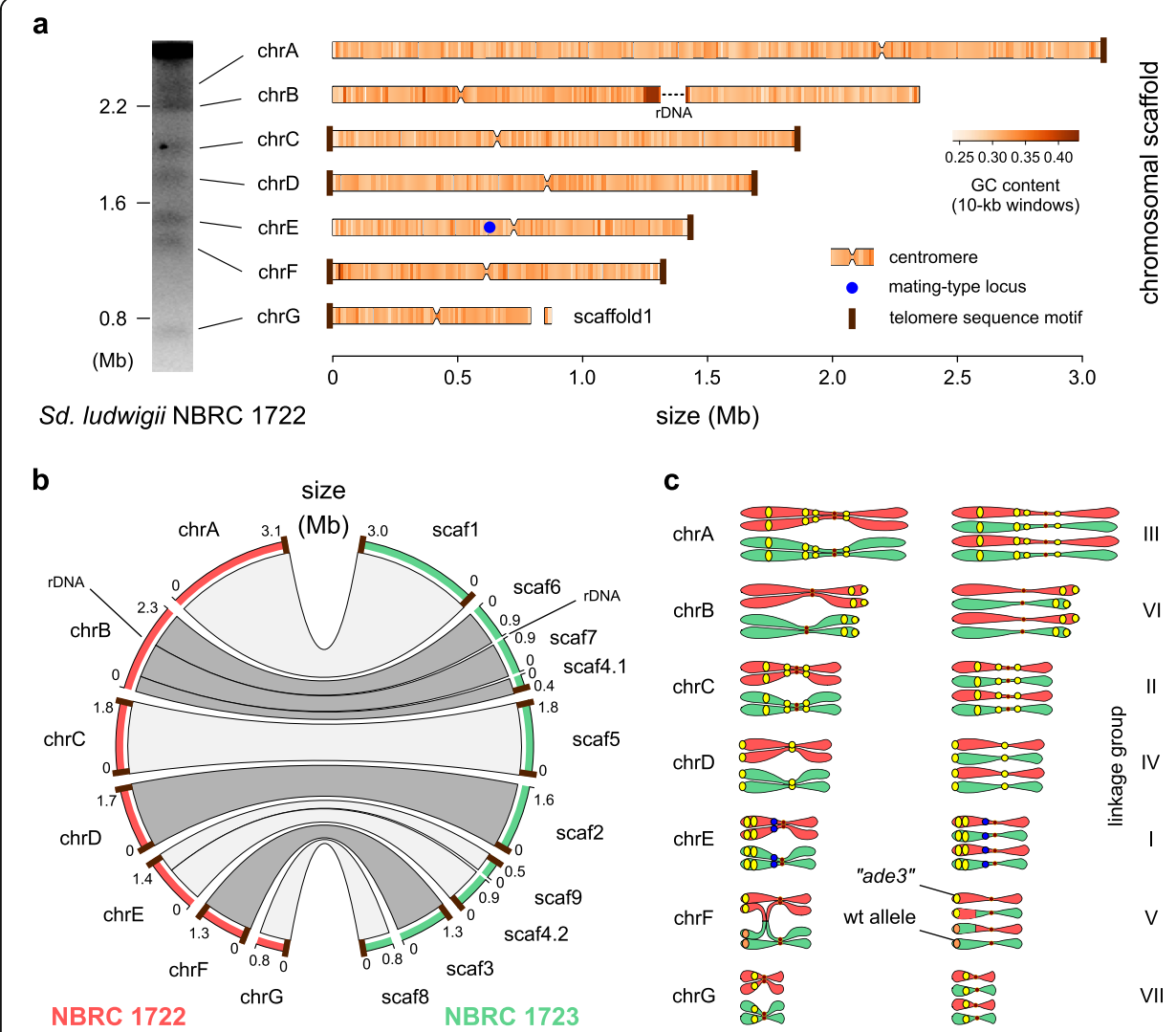

Fig. 2 Genome structure of Sd. ludwigii and mapping of previously used genetic markers. a The nuclear genome of the haploid reference strain NBRC 1722 is organized in 7 chromosomes, resolved as chromosomal bands on a PFGE gel (left) and depicted as bars (right). The only gap in the assembly (in chrB) corresponds to the internal part of the rDNA region, which is flanked by assembled rDNA repeats. b Genomic comparison of the Sd. ludwigii parental strains used in the previous genetic analyses. $\mathbf{c}$ The 7 previously defined $S d$. ludwigil linkage groups [12] were matched to the 7 chromosomes of the reference genome assembly, and 16 of the previously used markers were unambiguously mapped on the assembly (yellow circles). The MAT locus is shown as a blue circle (in chrE). One of the rare COs that were previously detected using tetrad analysis was tracked down to a reciprocal exchange event between the marker "ade3" (corresponding to the ADE4 gene) and the centromere of chrF, depicted here as an example

of sequence identity ( $95.3 \%$ on average). These findings exclude the possibility that the absence of COs could be due to major differences between homologous chromosomes.

Another possibility for the explanation of the extreme rarity of previously detected COs could be a non-random chromosomal distribution of the UV-generated genetic markers used in those analyses $[11,12]$. By combining the results of our gene prediction and annotation of the reference assembly with the phenotypic gene annotations of S. cerevisiae (www.yeastgenome.org), we identified the chromosomal positions of 16 out of the 24 used markers (Fig. 2c). This analysis revealed that all chromosomes were covered by markers, most of which were quite distant from the corresponding centromeres. Therefore, the previously used experimental setup [11, 12] appears sufficient for capturing the majority of COs in the largest part of the genome.

\section{The Sd. ludwigii meiotic gene machinery}

To gain insight into the genetic causes of the unusual meiotic behavior of $S d$. ludwigii, we manually refined the gene prediction and we curated the annotation of Sd. ludwigii genes 
based on the available S. cerevisiae dataset (www.yeastgenome.org). Gene prediction yielded a total of 5347 genes, of which 5031 are protein-coding ORFs. Among these genes, we identified homologs of 272 out of the 284 genes of S. cerevisiae with annotated functions in meiosis (Gene Ontology terms; www.yeastgenome.org), using protein sequence similarity and gene synteny as criteria (Fig. 3a; Additional file 4: Table S3). All genes that are required for wildtype levels of meiotic recombination in S. cerevisiae have homologs in Sd. ludwigii, with the only exception of MER1 [48] (Fig. 3a). This gene codes for a meiosis-specific splicing activator for introns in the genes AMA1, HFM1, REC107, and SPO22 (ZIP4), which function in chromosome pairing, meiotic recombination, and cell cycle regulation. Deletion of $M E R 1$ in $S$. cerevisiae abrogates the expression of these 4 target genes and causes major defects in meiotic

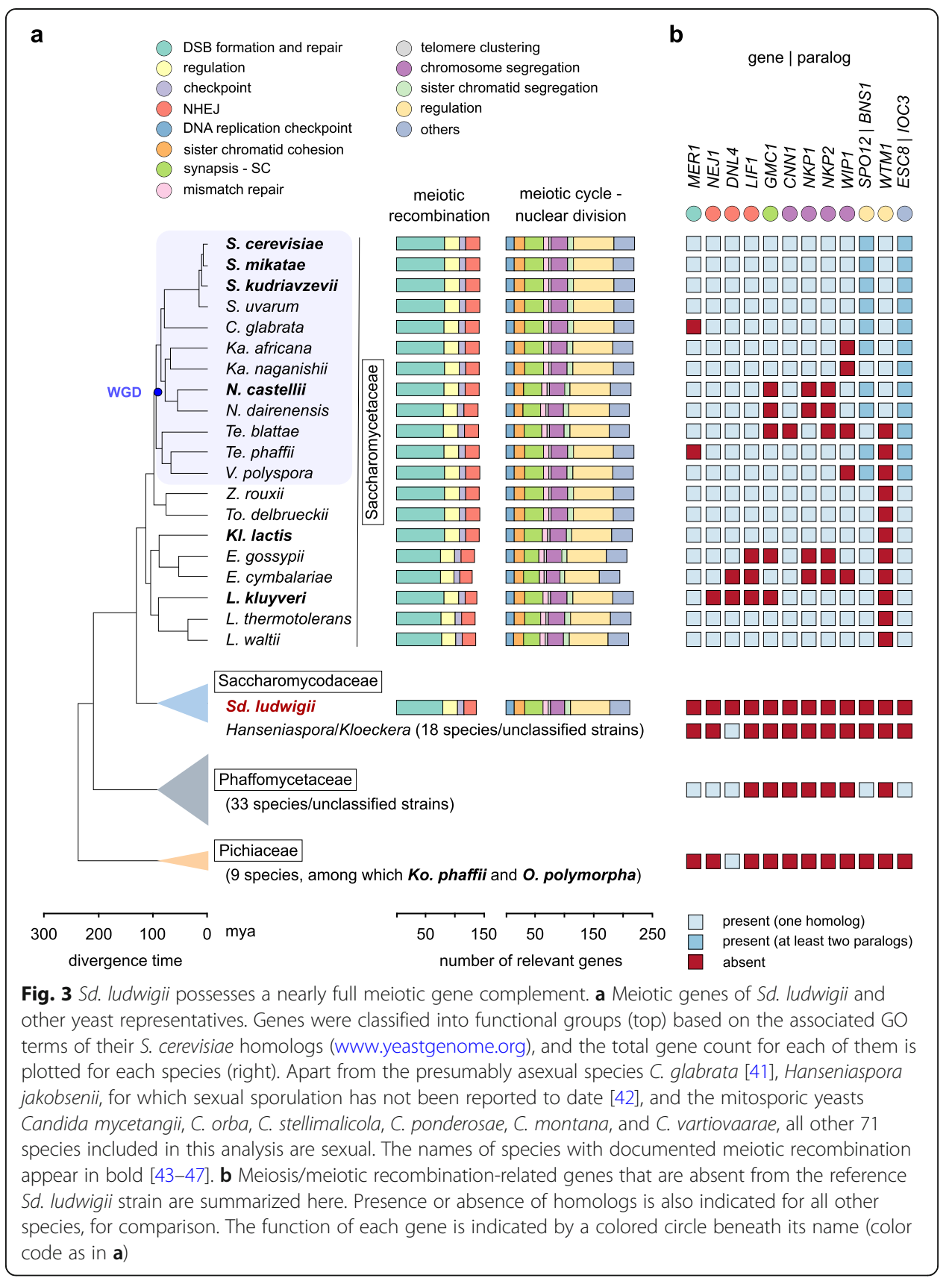


recombination and progression $[48,49]$. However, the Mer1 regulon of $S d$. ludwigii appears largely rescued, as no introns were detected in 3 of its target genes (AMA1, HFM1, and REC107), while SPO22 retains a predicted intron. By investigating the phylogenetic distribution of MER1, we confirmed its ancestral origin as syntenic homologs were identified in the distant families Phaffomycetaceae, Ascoideaceae, and Saccharomycopsidaceae (Figs. 1a, 3a). Therefore, its absence from Sd. ludwigii and the closely related Hanseniaspora species must be due to a secondary loss early in the evolution of their family (i.e., Saccharomycodaceae; Fig. 3a).

Apart from MER1, no homologs were detected in Sd. ludwigii for 11 additional genes with meiotic functions, namely NEJ1, DNL4, LIF1, GMC1, CNN1, NKP1, NKP2, WIP1, SPO12/BNS1 (paralogs in S. cerevisiae), WTM1, and ESC8/IOC3 (paralogs in S. cerevisiae) (Fig. 3b). In contrast to $M E R 1$, inactivation of any of these genes in S. cerevisiae does not abrogate meiotic crossing over. With the exception of DNL4, these genes are also absent from members of the Pichiaceae that are known to form COs in meiosis, such as Komagataella phaffii and Ogataea polymorpha [43, 44]. A phylogenetic analysis suggested that 7 of these genes (i.e., LIF1, NKP1, NKP2, CNN1, WIP1, GMC1, and WTM1) are present only in Saccharomycetaceae members, and thus have probably emerged after the separation of the $S d$. ludwigii lineage. Among the remaining 4 genes, DNL4 and NEJ1 (involved in non-homologous end joining, NHEJ [50]) are also missing from Lachancea kluyveri, which is capable of meiotic crossing over [45], while SPO12 [51] and ESC8 [52] have only minor and indirect roles in S. cerevisiae meiosis. Overall, our results demonstrate very limited meiotic gene loss and the retention in Sd. ludwigii of the essential gene machinery for meiotic recombination.

Eight of the putative $S d$. ludwigii meiotic proteins have little or no similarity to their S. cerevisiae homologs, and the identification of their genes was mostly based on synteny. These are MEI4, NDJ1, PSY3, SPO16, REC104, POL4, IML3, and HED1 (Additional file 4: Table S3). Using similarity searches with either the $S$. cerevisiae or $S d$. ludwigii protein sequences as queries, most of these genes could not be detected in the closely related Hanseniaspora species, which is consistent with extensive loss of genes involved in DNA repair, cell cycle regulation, and meiosis from this genus [53].

\section{Meiotic recombination components are required for Sd. ludwigii meiosis}

The presence of a nearly intact meiotic gene machinery suggests that these genes are functional in Sd. ludwigii. This is further supported by our finding that deletion of SPO11, which in other organisms initiates meiotic recombination by generating DNA DSBs, led to significantly reduced sporulation and spore viability (Fig. 4a), similarly to spo11 hypomorphs of S. cerevisiae [54, 55]. These results indicate an important function of this protein in meiosis. Since $S$. cerevisiae $\triangle$ spo11 mutants also exhibit defects in chromosome synapsis [10], we also deleted $S A E 2$, a regulator promoting the dsDNA endonuclease activity of Mre11 in the Mre11-Rad50-Xrs2 complex that operates downstream of Spo11 in DSB processing [56]. This led to a similar sporulation defect to that of the $\Delta$ spo11 strain (Additional file 2: Fig. S3), suggesting a role for Spo11 in catalyzing DSB formation in Sd. ludwigii meiosis. In order to confirm directly the occurrence of Spo11-dependent DSBs, we investigated the meiotic localization of Rad51, a recombinase that is involved in DSB repair [8]. Using an anti-Rad51 antibody, we detected 
a
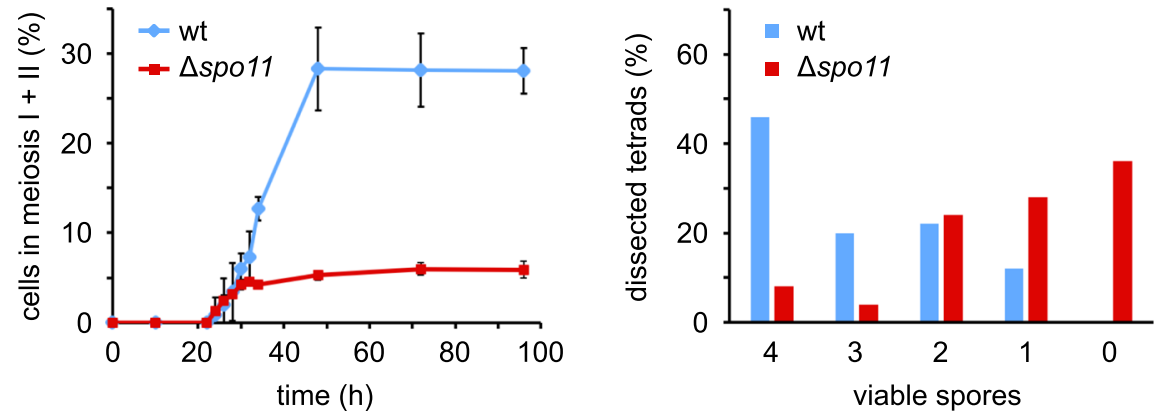

b
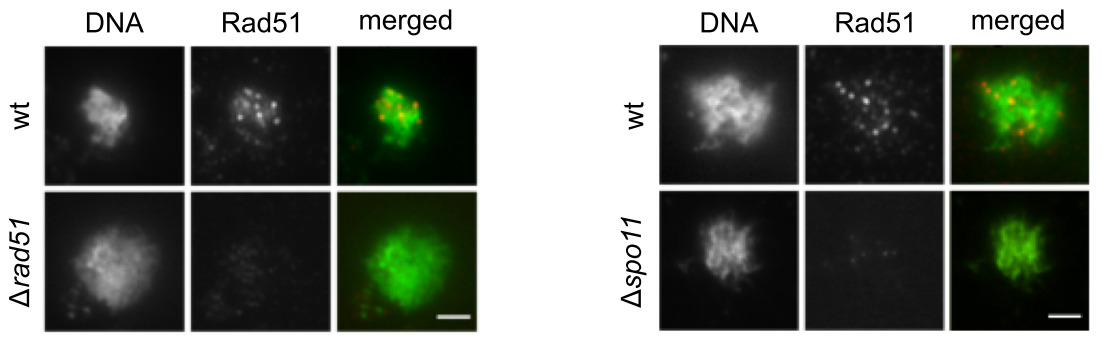

C

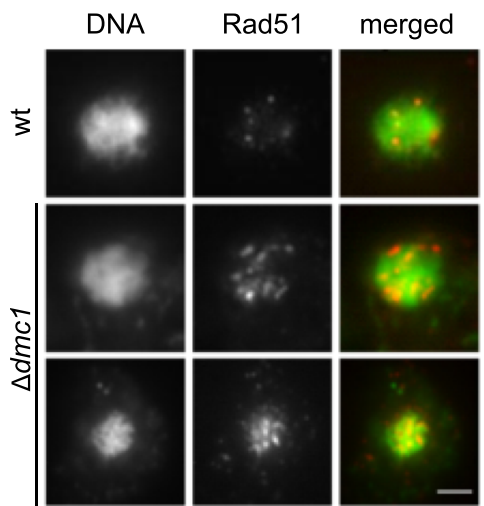

d

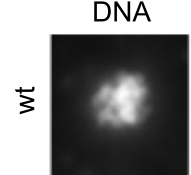

Rfa1

merged
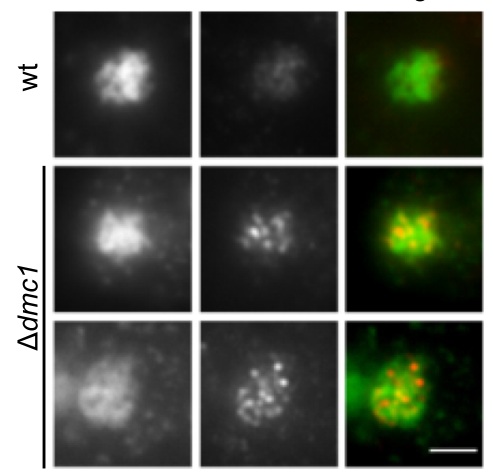

Fig. 4 Core meiotic recombination components are required for normal meiosis in Sd. ludwigii. a Meiotic time-course analysis of a homozygous $\Delta$ spo $11 \mathrm{Sd}$. ludwigii mutant in comparison to its wild type (left). After induction of meiosis, samples were withdrawn at the indicated time points and their cellular DNA content was stained with Hoechst 33258 to determine the fractions of binucleate (meiosis I) and tetranucleate (meiosis II) cells. Error bars: SD ( $n=3$ replicates for each strain). Spore viability was examined using tetrad dissection and viable colony counting (right). A total of 50 tetrads of each strain were analyzed. b-d Meiotic nuclear spreads of sporulating cells with the indicated genotypes (homozygous diploids). Cellular DNA was stained with Hoechst 33258. Immunostaining was performed using anti-Rad51 or anti-Rfa1 antibodies. Bars: 1 m

discrete foci in spreads of meiotic Sd. ludwigii chromosomes. No such foci were observed in $\Delta$ rad51 or $\Delta$ spo11 mutants, indicating the specificity of detection and the dependence of DSBs on Spo11 (Fig. 4b). We detected 5-12 foci per nucleus, which would be consistent with the formation of 1-2 Spo11-dependent DSBs per meiotic chromosome. Our results overall suggest that Spo11 is required for normal meiosis in $S d$. ludwigii, through the generation of meiotic DNA DSBs.

We also performed Rad51 immunostaining in meiotic spreads of $S d$. ludwigii cells deleted for $D M C 1$, a ubiquitous meiosis-specific recombinase involved in DSB repair as 
well as in promoting interhomolog interactions. In this case, we observed elongated, filamentous foci (Fig. 4c), which could be consistent with the facts that in S. cerevisiae the filaments of both proteins often co-occupy the same ends of meiotic DSBs [57], and absence of Dmc1 leads to persistent Rad51 foci, which accumulate and become less punctate with time [58]. Another conserved single-stranded (ss) DNA-binding protein that is involved in DNA replication, repair and recombination, is Rfa1. We detected expanded Rfa1 foci in $\Delta d m c 1$ meiotic spreads, whereas no foci were detected in wild-type spreads (Fig. 4d). This is congruent with the normally transient nature of ssDNAbinding activity of Rfa1, which is replaced by Dmc1, and the accumulation of ssDNA at DSBs in $\Delta d m c 1$ cells, similarly to S. cerevisiae [59]. Overall, formation of meiotic DSBs and their recombinational repair, mediated by key meiotic components, are required for normal meiosis in Sd. ludwigii, consistently with what is known from S. cerevisiae.

\section{High-resolution analysis of meiotic segregation in Sd. ludwigii}

Our findings suggest that meiotic recombination might still be occurring in $\mathrm{Sd}$. ludwigii, albeit with patterns that perhaps prevented detection by segregation analyses using only few markers. To address this, we performed a high-resolution genome-wide SNP segregation analysis of Sd. ludwigii meiosis (Fig. 5a; Additional file 2: Fig. S4a). We used as parents the reference strain and a spore that we isolated from a tetrad of a geographically distant strain (spore 122; Additional file 1: Table S1). Crosses between these two haploid strains frequently formed tetrads with 4 viable spores. Long-read sequencing and de novo assembly of the second parent's genome did not reveal significant karyotypic differences that could prevent pairing of homologous chromosomes in this cross (Fig. 5b). Variant calling between the two strains resulted in a total of 199,356 highquality SNPs, with an overall sequence divergence of $1.59 \%$, while the mean and median distances between adjacent SNPs were 62 and 16 bp, respectively. Notably, heterogeneity was observed in SNP density across the genome, with the higher-heterozygosity genomic compartments (i.e., chrC, chrF, chrG, and parts of chrA and chrB; $44.6 \%$ of the genome) exhibiting on average 3.3\% divergence (mean/median distance between SNPs: 38/16 bp), whereas the remaining, less divergent parts of the genome (i.e., chrD, chrE, and the remaining parts of chrA and chrB; $55.4 \%$ of the genome) displayed on average $0.2 \%$ sequence divergence, and $470 / 201$ bp between SNPs (mean/median distance) (details per chromosome are provided in Additional file 2: Fig. S4b).

Sequencing of all spores from 5 full tetrads from this cross followed by analysis of the SNP segregation patterns revealed complete absence of meiotic COs (Fig. 5c). The same was observed when we extended our analysis to 2 tetrads from another diploid strain (originating from the wild-type isolate CBS 5929; Additional file 1: Table S1; Additional file 2: Fig. S4a; Fig. S4b). These results confirm the previous genetic evidence that suggested extreme rarity of meiotic crossing over in $S d$. ludwigii [11, 12]. Among the 7 tetrads, we detected 2 independent single-marker NCOs (gene conversion tracts with 3:1 segregation patterns; Fig. $5 \mathrm{~d}$ ), which were validated by PCR amplification and sequencing. Their maximum tract lengths were 5.8 and $0.6 \mathrm{~kb}$, respectively (calculated based on the distance between their closest flanking SNPs with 2:2 segregation patterns). This suggests that at least some of the meiotic DSBs are processed by interactions that use the homologous chromosome as repair template without, however, 


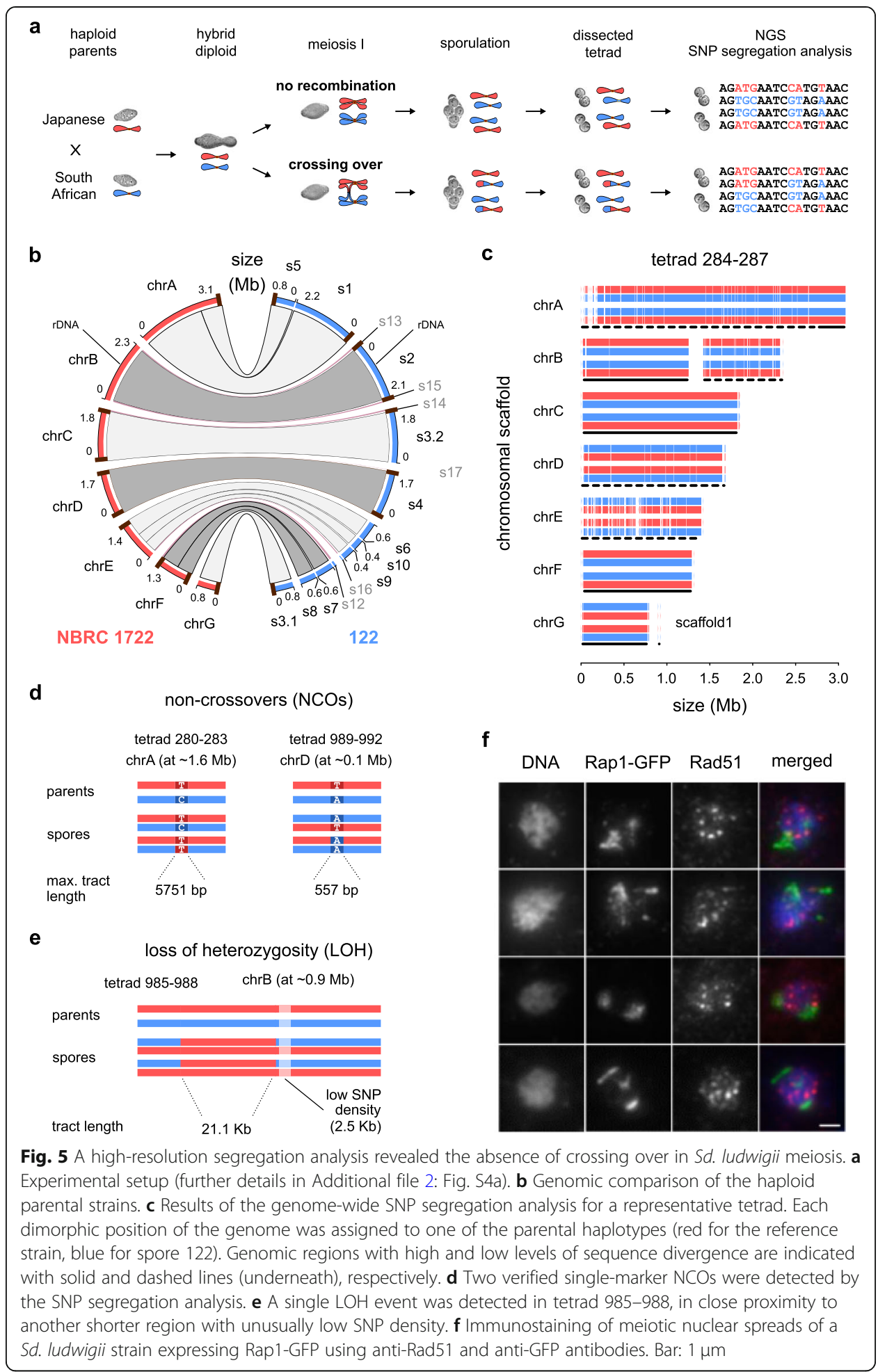

leading to $\mathrm{CO}$ formation. Finally, our analysis revealed a $21.1 \mathrm{~kb}-$ long loss-ofheterozygosity ( $\mathrm{LOH}$ ) tract (Fig. 5e), which could have resulted from a mitotic gene conversion event during diploid growth preceding sporulation. This $\mathrm{LOH}$ event occurred in close proximity to a $2.5 \mathrm{~kb}$-long region of distinctly lower SNP density than 
that of the flanking chromosomal regions (Fig. 5e), indicating that another ancient event had occurred in the same region.

High sequence divergence between homologous chromosomes is known to lower the probability of recombination, perhaps especially of class I interfering COs, due to the mismatch repair system that rejects recombination intermediates and redirects them towards NCOs or sister-chromatid recombination [60, 61]. This could probably impede recombination in the high-diversity genomic regions, but the low-heterozygosity parts, which constitute more than half of the hybrid genome, should not be affected, since their divergence levels are substantially lower than those used in previous yeast studies $[43,45,62,63]$. Despite the particularly high resolution of our analysis in the higherdiversity regions, we detected only 2 NCOs in total, which only leaves open the possibility of sister-chromatid repair.

Telomeric regions of chromosomes are highly repetitive (Additional file 2: Fig. S1b), and this could compromise the sensitivity of our method for the detection of COs in these regions [31, 43, 45, 62]. To investigate the possibility of crossing over in telomeric or telomere-proximal regions, we used meiotic chromosome spreads to compare the localization of GFP-tagged Rap1, a cytological marker of telomeres [64], and that of Rad51 foci as an indicator of meiotic DSBs [8]. If subtelomeric DSBs mediated chromosome interactions for meiosis I, we would expect to have at least one Rad51 dot per chromosome at or near a telomere. Since we only detect approx. 10 Rad51 foci per nucleus, we would expect to see more than half of the Rad51 dots colocalizing with Rap1. This was never the case (Fig. 5f) in 17 investigated nuclei. Rare colocalization of a single Rad51 dot with Rap1 could be expected to happen by chance, since Rap1 does not only localize to telomeres, but also to other chromosomal regions [65]. Therefore, initiation of recombination is not biased towards telomeres or adjacent regions in Sd. ludwigii, and the extreme suppression of crossing over appears to affect the entire genome.

\section{Search for signs of historical crossing over in Sd. ludwigii}

The unusual suppression of homolog interactions in Sd. ludwigii meiosis motivated us to search for signs of recombination by comparing divergent strains of international origin. For this, we sequenced 10 available haploid and diploid strains (Additional file 5: Table S4), two of which were found to be aneuploids $(2 n+1$ trisomies) by a read coverage analysis (Additional file 2: Fig. S5a). Variant calling and comparison to our reference strain revealed variable sequence divergence $(\sim 18,000-308,000$ SNPs $)$ and heterozygosity levels in diploids ( 1300-60,000 heterozygous SNPs) (Additional file 5: Table S4). Apart from the very divergent haploid strain PC99_R_1, sequence variation showed non-uniform patterns of distribution across the genome, being mostly restricted to particular chromosomes (spore 122) or, in most strains, to chromosomal segments (Additional file 2: Fig. S5b).

Phylogenetic analysis of all strains based on their genome-wide SNP content revealed the presence of a major cluster of 7 strains, and 4 more divergent ones (Fig. 6a). When we compared the dendrograms of individual chromosomes, we observed chromosomedependent topologies and distances for particular strains (Fig. 6a, b; Additional file 2: Fig. S5c). Our analyses revealed that chromosomes of strains with unusually unstable topologies differ significantly in SNP density from their genomic average (Additional 


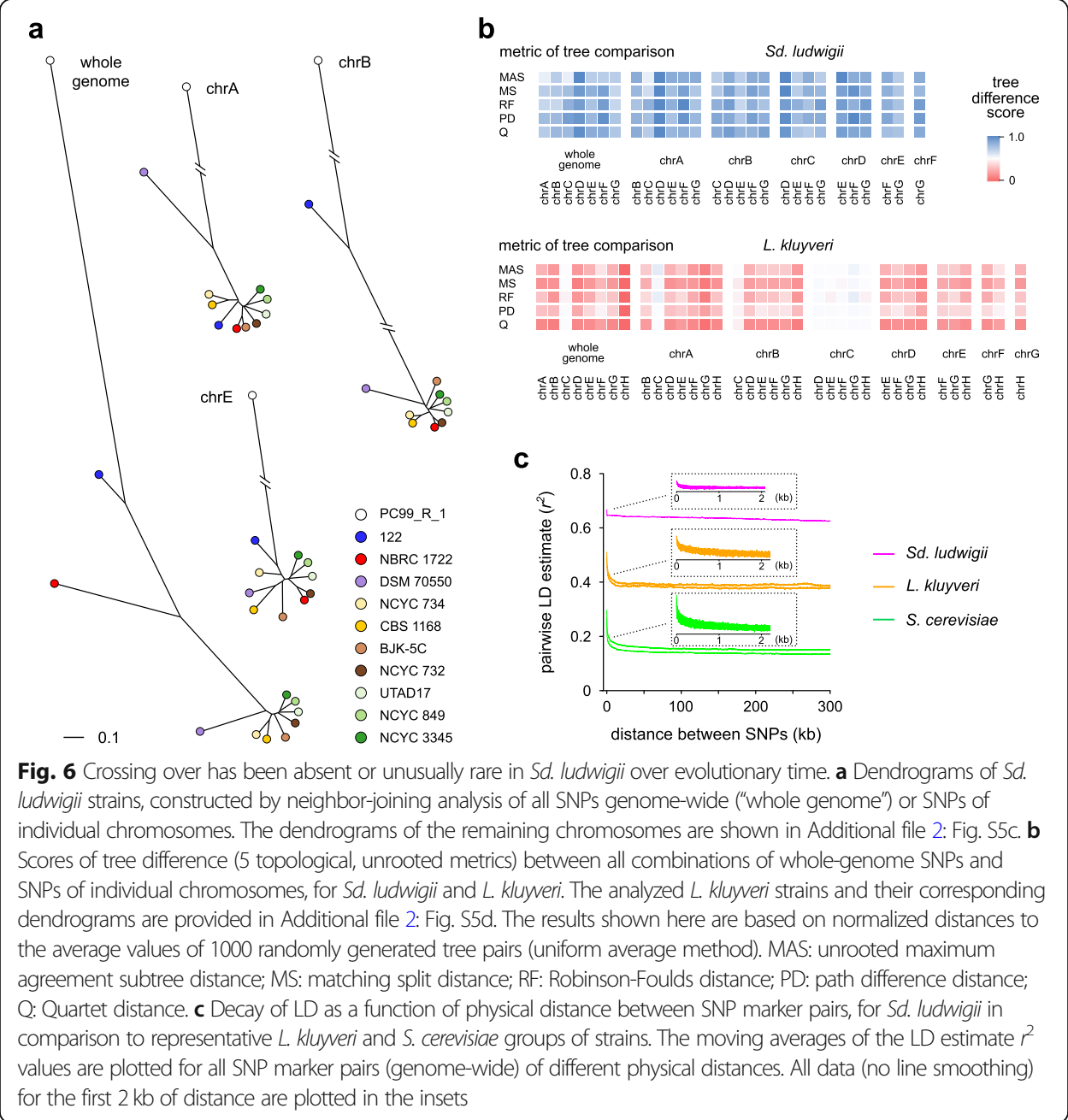

file 2: Fig. S5e). One possible explanation for these contrasting chromosome-specific signals could be that absence or rarity of meiotic recombination over an extended period of time has deprived chromosomes of the corresponding homogenizing effect, allowing them to accumulate SNPs independently from their homologs to a certain extent. We gained further support for this hypothesis by performing the same analysis in the yeast Lachancea kluyveri, a Saccharomycetaceae member with a similar genome size and chromosome number to Sd. ludwigii, but with demonstrated capability of meiotic recombination [45]. The analysis revealed very stable topologies in L. kluyveri, with all chromosomes yielding the same topology as the whole genome (Additional file 2: Fig. S5d, 5f; Fig. 6b).

Next, we performed a genome-wide linkage disequilibrium (LD) analysis in Sd. ludwigii, in comparison to L. kluyveri and S. cerevisiae, which are characterized by low and high meiotic recombination activity, respectively [45, 62]. Generation of LD decay curves, for which the LD estimate $r^{2}$ for each pair of SNPs is plotted versus their physical distance, revealed striking differences between the species (Fig. 6c). The overall levels of LD were unusually high in Sd. ludwigii, intermediate in L. kluyveri, and lowest in S. cerevisiae. Furthermore, LD decayed extremely fast in Sd. ludwigii, where it reached its plateau within the first $\sim 50 \mathrm{bp}$, in contrast to the more gradual decay of the 
2 other species, which is typical of recombining organisms [66, 67]. Four of the $S d$. ludwigii chromosomes exhibited even higher LD levels and faster decay than the genomic average (Additional file 2: Fig. S6a). To address the limitation of the small sample size in these analyses (due to the limited availability of wild-type $S d$. ludwigii strains), we always compared groups of the same size (11 strains for all species), and we also compared multiple groups of randomly selected strains of L. kluyveri and S. cerevisiae. Curves of LD decay were quite stable and the observed differences between species were independent of the particular group considered (Additional file 2: Fig. S6b). These results are consistent with the hypothesis of absent or very rare meiotic recombination in Sd. ludwigii over evolutionary time, which would decrease the overall LD levels and decelerate its decay.

\section{Mutation rate and genomic evolution in Sd. ludwigii}

A hypothetically elevated mutation rate could be a compensatory solution for the generation of genetic diversity in Sd. ludwigii in the absence of meiotic recombination, or even serve as a driver of achiasmate meiosis [27]. Furthermore, we reasoned that a significant AT bias of spontaneous mutations could explain the particularly low GC content of the Sd. ludwigii genome (Additional file 2: Fig. S2a). We investigated these hypotheses directly by a mutation accumulation analysis in 3 founder Sd. ludwigii strains: our haploid reference strain, a corresponding homozygous diploid strain, and the hybrid diploid strain used in our SNP segregation experiment (Table 1; Additional file 1: Table S1). We adopted a single-cell population bottlenecking regime of growth on agar plates to evolve 60 independent mutation accumulation lines for $\sim 2000$ generations each. Such an experimental setup ensures fixation of the majority of spontaneous mutations and minimizes elimination of non-lethal deleterious mutations due to selection [68]. Sequencing and genome-wide analysis revealed a total of 186 line-exclusive single-nucleotide mutations (SNMs) (Additional file 6: Table S5). This corresponds to an overall base-substitutional mutation rate $\left(\mu_{\mathrm{bs}}\right)$ of $5.70 \times 10^{-11}-1.20 \times 10^{-10}$ mutations per base per generation, for the 3 strain genealogies (Table 1). These rates, which are in line with the rate of another recently analyzed $S d$. ludwigii diploid strain [69] $\left(7.3 \times 10^{-11}\right)$, fall within the range of unicellular eukaryotes, and they are even slightly

Table 1 Mutation accumulation analysis. The base-substitutional mutation rate $\left(\mu_{\mathrm{bs}}\right)$, its Poisson 95\% confidence interval (Cl), the AT bias (weighted by genomic nucleotide composition), and the transition:transversion ratio ( $\mathrm{Ts}$ :Tv) of spontaneous single-nucleotide mutations are provided for

\begin{tabular}{|c|c|c|c|c|c|c|c|c|}
\hline Strain & $\begin{array}{l}\# \\
\text { generations } \\
\text { (average) }\end{array}$ & $\begin{array}{l}\# \\
\text { mutations } \\
\text { (\# lines) }\end{array}$ & $\begin{array}{l}\mu_{\mathrm{bs}}(/ \mathrm{site} / \\
\text { generation) }\end{array}$ & $\begin{array}{l}\text { Poisson } \\
95 \% \mathrm{Cl}\end{array}$ & $\begin{array}{l}\text { AT } \\
\text { bias }\end{array}$ & $\begin{array}{l}\text { Mutation-driven } \\
\text { equilibrium GC } \\
\text { content }\end{array}$ & $\begin{array}{l}\text { Observed } \\
\text { GC } \\
\text { content }\end{array}$ & $\begin{array}{l}\text { Ts: } \\
\text { Tv } \\
\text { ratio }\end{array}$ \\
\hline $\begin{array}{l}\text { Haploid } \\
\text { (NBRC } \\
1722 \text { ) }\end{array}$ & 2037 & $85(30)$ & $1.20 \times 10^{-10}$ & $\begin{array}{l}9.62 \times \\
10^{-11}-1.49 \\
\times 10^{-10}\end{array}$ & 2.92 & 0.255 & 0.296 & 1.18 \\
\hline $\begin{array}{l}\text { Isogenic } \\
\text { diploid } \\
\text { (YLFP17-4) }\end{array}$ & 1920 & $48(19)$ & $5.70 \times 10^{-11}$ & $\begin{array}{l}4.20 \times \\
10^{-11}-7.55 \\
\times 10^{-11}\end{array}$ & 5.85 & 0.146 & 0.296 & 1.53 \\
\hline $\begin{array}{l}\text { Hybrid } \\
\text { diploid } \\
\text { (YLFP188- } \\
\text { 1) }\end{array}$ & 2016 & $53(11)$ & $9.55 \times 10^{-11}$ & $\begin{array}{l}7.16 \times \\
10^{-11}-1.25 \\
\times 10^{-10}\end{array}$ & 2.63 & 0.276 & 0.296 & 0.96 \\
\hline
\end{tabular}


lower than those of other yeast species [69, 70]. Therefore, mutation rate in Sd. ludwigii is not unusually elevated. Similarly, the overall transition-to-transversion (Ts:Tv) ratio (1.21 in Sd. ludwigii) is comparable to the values reported for S. cerevisiae and expected for organisms with no cytosine methylation [70].

The $S d$. ludwigii genome has a remarkably low GC content. We determined that, similarly to the majority of eukaryotes, Sd. ludwigii has a strong AT bias of spontaneous mutations, with SNMs that convert GC to AT occurring on average 3.6 times more frequently than SNMs in the opposite direction (Table 1). Based on this, the theoretical equilibrium GC content of the $S d$. ludwigii genome should be $23.3 \%$, if it were determined by spontaneous mutations alone. In these calculations, we excluded repeat regions ( 7\% of the genome), since they are difficult to analyze and are associated with a high indel formation frequency [71]. The observed genomic GC content of the analyzed genomic fraction was $29.6 \%$, which cannot be explained by the effect of spontaneous mutations only. Therefore, balancing forces in the opposite direction, such as selection on GC or GC-biased gene conversion [70], are probably also present in Sd. ludwigii.

\section{Mitotic LOH in Sd. ludwigii}

The striking rarity of homolog interactions in $S d$. ludwigii meiosis prompted us to investigate the levels of mitotic homologous recombination, to understand whether the observed strong suppression is specific to meiosis. Further analysis of the 11 mutation accumulation lines of the hybrid diploid strain (Table 1) revealed a total of $22 \mathrm{LOH}$ events (Additional file 7: Table S6), half of which occurred in chrA. Chromosome A is the largest chromosome (representing $\sim 25 \%$ of the $S d$. ludwigii genome). Whether this relative enrichment of events in this chromosome explains its lower SNP density compared to other chromosomes (Additional file 2: Fig. S4b) is unclear. Similarly, non-uniform distributions of LOH events have been observed in $S$. cerevisiae [72, 73]. Among the detected events, we identified one long terminal event (in chrC; $\sim 183 \mathrm{~kb}$ ), which could be attributed to either a mitotic crossover or a break-induced replication (BIR) event [74]. The remaining events were shorter interstitial LOH regions with a maximum length of $12.5 \mathrm{~kb}$ (Additional file 7: Table S6). These may be associated with mitotic crossing over or gene conversion $[72,73,75]$. The total genomic rate of LOH events was 9.9 $\times 10^{-4}$ events per generation, which is moderately lower than the 3-10 times higher frequencies reported for S. cerevisiae [72, 73]. Therefore, the core machinery for repair of spontaneous DSBs during the vegetative life phase of $S d$. ludwigii is present and functional. The extreme suppression of recombination is, therefore, limited to meiosis.

\section{Discussion}

Repair of meiotic DSBs during prophase I can result in chiasmata that promote accurate segregation of homologous chromosomes and enable genetic exchanges between them $[8,9,13]$. Meiotic cells preferentially rely on homologous recombination, which can repair a subset of these DSBs to form COs, whereas the remainder lead to NCOs or use the sister chromatid as repair template [76, 77]. The pathway choice decision is controlled by multiple cytological factors [78] and can be subject to evolutionary selection. Our study revealed that Spo11-mediated introduction of DSBs is required for normal meiosis in Sd. ludwigii. Given that immunostaining of Rad51 for visualizing DSBs may underestimate their number [58], we hypothesize that the observed 5-12 Rad51 
foci correspond to at least one to two DSBs per chromosome in Sd. ludwigii meiosis. The demonstrated involvement of Dmc1 in DSB processing further suggests that at least a fraction of these DSBs are processed through homolog-templated repair pathways $[8,9,13]$, but with a strong bias towards NCOs as suggested by the absence of COs and the detection of two NCOs in our meiotic SNP segregation analysis. Since NCOs typically involve short gene conversion tracts (usually 1-2 kb in S. cerevisiae [62, 79]), more such events might have occurred in our analyzed tetrads below the resolution limit of the analysis. The total absence of crossing over throughout the genome corroborates the hypothesis of Yamazaki and coworkers, who proposed that crossing over as an obligatory feature of meiosis I might be absent from $S d$. ludwigii meiosis $[11,12]$. Considerable involvement of NHEJ in the repair of meiotic DSBs is unlikely, given that NHEJ is generally suppressed during meiosis [80, 81], and Sd. ludwigii misses four components of the major NHEJ pathway.

The level of heterozygosity present in individuals and, therefore, the extent of genetic diversity in populations are largely dependent on mating strategies. Frequent intratetrad mating, in particular, preserves high levels of heterozygosity in parts of the genome (Fig. 7a), ensures efficient purging of deleterious mutations, and provides fitness advantages $[24,26,27,82]$. Suppression of recombination in organisms that often engage in intratetrad mating could be beneficial for their evolution by extending the preservation of heterozygosity to larger parts of their genomes [25] (Fig. 7b). Our findings support the hypothesis that achiasmate meiosis might have evolved in the Sd. ludwigii lineage through mutual selection between suppression of meiotic recombination and frequent intratetrad mating, which is its predominant mating behavior [34, 83]. Low recombination rates could have arisen through the accidental loss of an important meiotic gene, e.g., MER1 [49], which could have spread to fixation possibly due to genetic hitchhiking by suppressing recombination along the entire chromosomes. The need to overcome

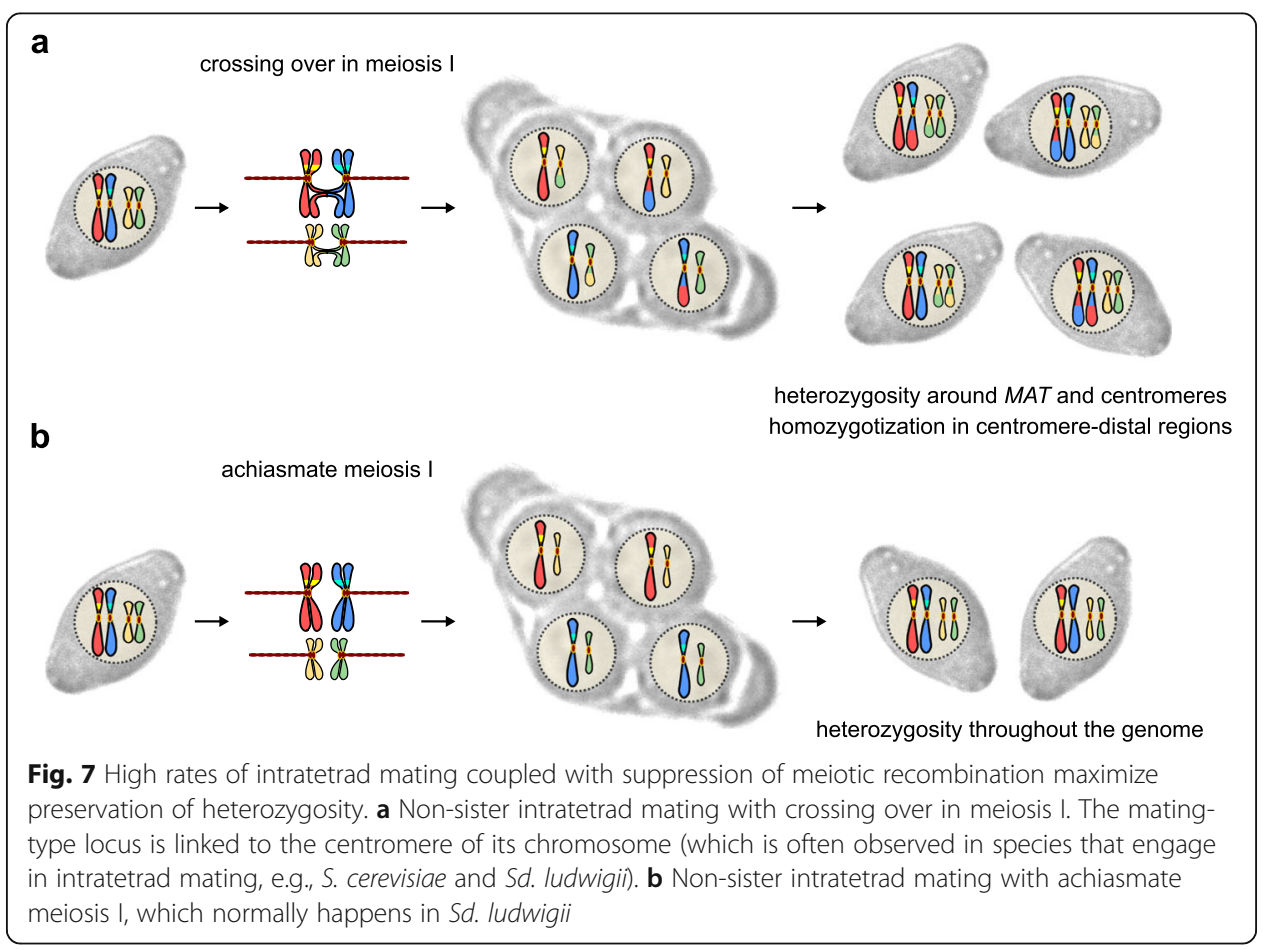


the negative consequences of chromosome non-disjunction due to the loss of crossing over could have driven the selection of mechanisms or behaviors that alleviate the problem of inviable spores due to chromosomal mis-segregation. This organism appears to be avoiding this risk by enforcing intratetrad mating in its life cycle through the development of robust interspore bridges that keep non-sister spores tightly linked together in its asci $[34,83,84]$. Mating with non-sister meiotic siblings would restore a full diploid chromosome complement (Fig. 7b) even in the case of aneuploid individual spores, which are still likely capable of mating [27, 85]. Absence of recombination would prevent the exchange of mating types between non-sister chromatids in meiosis I and, therefore, the requirement for opposite mating types would lead to full reconstitution of the parental diploid genomic content upon mating. In this scenario, the duet of suppressed recombination and frequent intratetrad mating could have persisted because of the efficient preservation of heterozygosity and the fitness advantages that this could offer, according to previous research [25-27].

Further support for our hypothesis that loss of MER1 could have mediated the extreme suppression of meiotic crossing over may be provided by the finding that one of its splicing targets, i.e., SPO22 (ZIP4), appears to retain an intron in Sd. ludwigii. This gene codes for a synapsis initiation complex component that is required for proper SC formation, chromosome synapsis, and class I (interfering) COs in S. cerevisiae [86]. Although Zip4 homologs of other organisms (A. thaliana, rice, and mouse) have only minor roles in the completion of synapsis, the important Zip4 function in interfering crossing over appears conserved [87-89]. Therefore, potential dysregulation of Zip4 due to the loss of Mer1 from Sd. ludwigii could lower its crossing over levels, or even cause their absence in case the class II CO pathway is not functional in this species. In accordance with this hypothesis, our preliminary investigations of $S d$. ludwigii meiotic proteins revealed the following: (i) markedly less elongated SC structures than in S. cerevisiae, possibly indicating defects in Zip1 polymerization, which in S. cerevisiae depends on Spo22/Zip4 [86], and (ii) while deletion of msh4 (class I CO formation [90]) led to remarkably defective meiosis, absence of Mms4 (class II crossing over [91]) had no effect on spore viability, which would be consistent with the idea that the class II pathway may be unimportant in Sd. ludwigii meiosis.

Another possible strategy that is often observed in Sd. ludwigii strains [92] is the completion of meiosis with only one division, which leads to two-spore asci with diploid spores. Secondary loss of SPO12, which is absent from Sd. ludwigii, might have helped to achieve this and prevent a meiotic arrest in cell cycle progression, since natural S. cerevisiae strains lacking SPO12 and SPO13 exhibit the same behavior [93].

The establishment of a lifestyle that successfully copes with the lack of meiotic recombination does not exclude the possibility of rare outbreeding [84] that would occasionally permit intermixing of chromosomes, as was observed in our population analysis (Fig. 6a; Additional file 2: Fig. S6c). In addition, the species seems not to have completely abolished its ability to rarely recombine its genome $[11,12]$, which explains the preservation of the corresponding gene arsenal, and the partial rescuing of its Mer1 regulon. Those processes would require the maintenance of chromosomal structural stability, without which the accumulation of gross rearrangements in homologs could hinder synapsis in meiosis I and preclude recombination. The loss of the classical NHEJ pathway from the Sd. ludwigii lineage (Fig. 3) could contribute to the preservation of 
genomic collinearity between its intra-specific lineages. Evidence from the older marker segregation studies [11, 12] suggested, indeed, that meiotic crossing over could still occur in Sd. ludwigii, but at extremely low frequencies (i.e., in $0.9 \%$ of their analyzed tetrads). Even if one assumes that the low resolution of their experiments led to a significant underestimation of that frequency, our higher-resolution data and the comparison with other yeasts clearly demonstrate that COs are extremely suppressed in $S d$. ludwigii meiosis: analysis of its 7 tetrads yielded no COs, in comparison to $\sim 630$ expected COs in S. cerevisiae [62], 315 in S. pombe [94], 175 in K. phaffii [43], and 125 in L. kluyveri [45].

Recombination, genomic GC content, and mutation rate are thought to be linked, although the causality of their relationships is still not fully understood [70, 95]. We performed a mutation accumulation analysis to investigate the reason for the particularly low genomic GC level in Sd. ludwigii. Its mutation rate falls within the normal range of related eukaryotes [70]. On the other hand, a strong AT bias of spontaneous mutations-similarly to many other organisms $[70,96]$-in conjunction with the presumably low relevance of GC-biased gene conversion $[68,70]$ due to the rarity of recombination, provides an explanation for the unusually low GC content of its genome. Balancing forces in the opposite direction, such as selection on GC [97] and temporary or conditional changes of the mutation rate and/or bias [98], must also act, given that the expected equilibrium genomic GC level would be $6.3 \%$ lower than the actual one if it were determined solely by the mutational load.

Loss of heterozygosity has been suggested to offer rapid evolutionary solutions for phenotypic diversification and adaptation, by exposing recessive beneficial alleles or alleviating negative epistasis of alleles in the heterozygous state [73, 99, 100]. Such adaptive flexibility could be very important for $S d$. ludwigii, which appears to suppress recombination of its genome. Our results indicate that $\mathrm{LOH}$ events, often resulting from mitotic COs or NCOs, occur in Sd. ludwigii at frequencies that are significantly higher than the base-substitution mutation rate. Suppression of recombination in this species is, therefore, limited to meiosis, and LOH could be an important mechanism for its adaptive potential.

The unique meiotic behavior of $S d$. ludwigii renders the functional dissection of its meiotic mechanisms and their regulation interesting goals of future research. Investigation of ZMM proteins that provide links between the SC, which facilitates segregation of meiotic chromosomes in many organisms [9], and repair of meiotic DSBs [101, 102], as well as characterization of components that regulate homolog bias and pathway choice $[9,78,103]$, could provide useful insights. Furthermore, an interesting subject could be the fast evolving meiotic proteins that were detected in Sd. ludwigii, such as Ndj1 and Spo16 that are involved in the regulation of CO formation and distribution in S. cerevisiae $[104,105]$. However, further functional investigations would require the development of $S d$. ludwigii strains with higher sporulation rates and better synchronization of meiosis I than the currently available ones.

\section{Conclusions}

Our study demonstrates that the high rate of intratetrad mating in the yeast species Saccharomycodes ludwigii is coupled with extreme suppression of meiotic crossing over throughout its genome and that this behavior has persisted over evolutionary time. We 
propose that this combination ensures adaptive genome evolution in a trajectory that requires only minimal levels of meiotic recombination. Therefore, $S d$. ludwigii provides a new paradigm and an excellent natural forum, in which to study experimentally the evolution of recombination rates and their influence on genome evolution.

\section{Methods}

\section{Yeast strains-strain construction}

All strains used in this study are listed in Additional file 1: Table S1. They were incubated at $30^{\circ} \mathrm{C}$ (with shaking of liquid cultures at $230 \mathrm{rpm}$ ) for vegetative growth or at $23{ }^{\circ} \mathrm{C}$ for sporulation. All plasmids are provided in Additional file 8: Table S7 [106109]. The E. coli strain $\mathrm{DH} 5 \alpha$ was used for their maintenance and propagation. All DNA oligonucleotides used for plasmid/strain construction are listed in Additional file 9: Table S8. Gene deletion and tagging were performed using the PCR targeting method [108]. A lithium acetate (LiAc) and heat shock-based protocol was optimized and used to transform Sd. ludwigii. Detailed methods for mating, sporulation, tetrad dissection, transformation, and strain construction, as well as for pulsed-field gel electrophoresis (PFGE), transmission electron microscopy (TEM), and ploidy determination using flow cytometry, can be found in the Additional file 10: Supplementary detailed methods [11, 12, 110-143].

\section{Immunostaining of meiotic spreads}

Spreads of $S d$. ludwigii meiotic nuclei were prepared from sporulating cells in meiotic time courses using the procedure previously described for S. cerevisiae [144] with some modifications. The detailed protocol is provided in the Additional file 10: Supplementary detailed methods. The primary antibodies used were as follows: rabbit anti-ScRfa1 (gift from E. Mancera), sheep anti-GFP (generated in our lab) at 1:500 dilution each, and rabbit anti-ScRad51 (gift from A. Shinohara) at dilution 1:1000. The latter antibody [145] was used for detection of discrete Rad51 foci, while the anti-GFP antibody was used for localization studies of GFP-tagged Rap1 in spreads of meiotic nuclei. The secondary antibodies used were as follows: Alexa Fluor 488-conjugated donkey anti-rabbit IgG (Dianova), Cy2-conjugated donkey anti-sheep IgG (Dianova) and Cy3-conjugated donkey anti-rabbit IgG (Dianova), at 1:500 dilution each.

\section{Mutation accumulation experiment}

Three Sd. ludwigii strains were used for the mutation accumulation study: the haploid reference strain NBRC 1722, the nearly isogenic diploid strain YLFP17-4 (homozygous diploid with the reference strain's background), and the hybrid diploid strain YLFP1881 (heterozygous diploid) (Additional file 1: Table S1). Clonal stocks of these parental strains were streaked on YPD plates, and randomly selected single colonies of each one served as founders of independent mutation accumulation lines (MALs). Spontaneous mutations were accumulated in 30,19, and 11 MALs of the haploid, the homozygous and the heterozygous diploid strain, respectively, while they were growing under favorable conditions (YPD medium, at $30^{\circ} \mathrm{C}$ ). Each MAL was passed through a single-cell population bottleneck every $48 \mathrm{~h}$ by picking a random single colony and streaking it to single colonies on fresh YPD plates again. The plates were pre-marked with a target, 
and the single colony closest to the target was picked for each cycle, to ensure random colony selection. The experiment was performed for a total of 96-97 cycles, which corresponds to 2037 generations for the haploid, 1920 generations for the homozygous diploid, and 2016 generations for the heterozygous diploid strain (each cycle corresponds to 20 generations for the homozygous diploid strain or 21 for the two other strains, as determined by resuspending 10 independent colonies of each one in water and counting cells using a Neubauer-improved hemocytometer, at the beginning of the experiment).

\section{Total DNA extraction from Sd. ludwigii}

Liquid Sd. ludwigii cultures in YPD medium (early stationary phase) were processed for DNA extraction using the Genomic-tip 20/G (mini prep, starting with approx. $10^{8}$ cells) or 100/G (midi prep, $10^{9}$ cells) kit (QIAGEN), according to the manufacturer's recommendations. An additional washing step with buffer $\mathrm{QC}$ was performed before the final elution step. To recover DNA following isopropanol precipitation, spooling with a pipette tip was performed. A washing step was performed using $500 \mu \mathrm{l}$ of ice-cold $70 \%$ ethanol. Following centrifugation $\left(13,000 \mathrm{rpm}\right.$ for $10 \mathrm{~min}$ at $\left.4{ }^{\circ} \mathrm{C}\right)$, ethanol was completely removed, the sample was air-dried at room temperature for $10 \mathrm{~min}$, and DNA was then resuspended in $100 \mu \mathrm{l}$ (mini prep) or $200 \mu \mathrm{l}$ (midi prep) TE buffer (pH 8.0). To further improve the quality of the isolated DNA, $100 \mu \mathrm{l}$ of each DNA sample were further processed using the DNeasy PowerClean Pro Cleanup kit (QIAGEN), according to the manufacturer's instructions. Total DNA was finally eluted in $50 \mu \mathrm{l}$ of elution buffer $(10 \mathrm{mM}$ Tris- $\mathrm{HCl}, \mathrm{pH} 8.0)$ and stored at -20 or $-80^{\circ} \mathrm{C}$. Alternatively, total DNA was isolated from $\mathrm{Sd}$. ludwigii using the phenol-chloroform extraction-based protocol that is provided in the Additional file 10: Supplementary detailed methods.

\section{Next-generation sequencing (NGS)}

\section{Illumina sequencing}

For sequencing on an in-house Illumina NextSeq 550 instrument, libraries were prepared using the NEBNext Ultra II FS DNA Library Prep Kit for Illumina (New England Biolabs), according to the manufacturer's recommendations. Enzymatic fragmentation of DNA was controlled to generate fragment size distributions in the range of 300-500 bp. For PCR enrichment of adaptor-ligated DNA, six or seven amplification cycles were used for samples with higher and lower starting amounts of DNA, respectively. Multiple DNA samples were barcoded with unique dual indices using the NEBNext Multiplex Oligos for Illumina (96 Unique Dual Index Primer Pairs) (New England Biolabs) and pooled at equimolar concentrations. The NEBNext Library Quant Kit for Illumina (New England Biolabs) was used for the qPCR-based quantification of the pooled library, which was then loaded on a high-output flow cell (NextSeq 500/550 High Output Kit v2.1, 300 cycles; Illumina) for sequencing in paired-end mode $(2 \times 150 \mathrm{bp})$.

Alternatively, Illumina sequencing was performed on MiSeq and HiSeq 2000 instruments, at the EMBL Genomics Core Facility (Heidelberg, Germany), in paired-end mode $(2 \times 150$ and $2 \times 100 \mathrm{bp}$ for MiSeq and HiSeq systems, respectively). Sequencing libraries were prepared by the Deep Sequencing Core Facility (University of Heidelberg, Germany). Briefly, mechanical shearing using a Covaris ultrasonicator was used for DNA fragmentation to a size range of 300-500 bp. The NEBNext Ultra II DNA Library 
Prep Kit for Illumina (New England Biolabs) was used for library preparation, and eight PCR cycles were performed for amplification of adaptor-ligated DNA.

\section{Pacific Biosciences SMRT (single-molecule real-time) sequencing (PacBio)}

For the generation of the de novo genome assembly of our reference Sd. ludwigii strain, we used PacBio sequencing, which was performed at GATC Biotech AG (now Eurofins Genomics). Briefly, library preparation involved DNA fragmentation, size selection (using a BluePippin instrument; Biozym Scientific), DNA end repair and adaptor ligation, and annealing of the primer and the polymerase. The PacBio library was then sequenced on 2 SMRT cells in a PacBio RS II instrument (run mode: 240 min. movie) using the P6-C4 chemistry. This yielded a total of $2.52 \mathrm{~Gb}$ of pass-filter sequence data, which corresponds to an average genomic sequencing depth of approx. 200x.

\section{Oxford Nanopore Technologies sequencing (ONT)}

Generation of genome assemblies of the other Sd. ludwigii strains was based on ONT sequencing on a MinION sequencer. The Ligation Sequencing Kit (for 1D experiments; SQK-LSK109) was used for library construction, starting from $1 \mu \mathrm{g}$ of total DNA of each sample, and different samples were barcoded using the Native Barcoding Expansion 1-12 (PCR-free) kit (both kits were purchased from Oxford Nanopore Technologies) and pooled for multiplexed sequencing (according to the manufacturer's instructions). No fragmentation of the input DNA was performed. The sequencing libraries were loaded on R9.4.1 flow cells and sequenced on a MinION device. Each strain was sequenced until the desired amount of sequencing data was gathered, corresponding to average genomic sequencing depths of approx. 30-40×.

\section{Genome assembly}

The de novo genome assembly of the reference Sd. ludwigii strain NBRC 1722 was based on high-coverage PacBio sequencing data. A total of 193,117 reads (average genomic sequencing depth of approx. 200x) with mean length 13,041 bp and mean $q$-score 0.827 passed the filter (SMRT Portal, Pacific Biosciences). The Celera Assembler [146] and the Quiver [147] tool in the HGAP.3 pipeline (Hierarchical Genome Assembly Process) within SMRT Analysis v2.3.0 (Pacific Biosciences) were used for genome assembly and consensus polishing, respectively. A part of the mitochondrial genome was identified using BLAST + v2.8.0 [120] and removed from the nuclear assembly. Subsequently, we used an Illumina MiSeq $2 \times 150$-bp paired-end read dataset generated from the same DNA sample for further polishing of the assembly using Pilon [148] (version 1.22 ), to generate the final assembly version (with an N50 value of $1,848,403 \mathrm{bp}$ ).

The de novo genome assemblies of strains 122 and NBRC 1723 were produced using ONT sequencing. We generated 93,607 filter-passing reads (average genomic sequencing depth of approx. $33 \times$ ) with an N50 read length of $6.89 \mathrm{~kb}$ and a mean $q$-score of 13.242 for strain 122, and 102,478 reads (average genomic depth of approx. $42 \times$ ) with an N50 read length of $7.76 \mathrm{~kb}$ and a mean $q$-score of 13.586 for strain NBRC 1723. The base-caller Guppy (v2.3.5; Oxford Nanopore Technologies) with the high-accuracy model was used for basecalling, and Porechop (v0.2.4; https://github.com/rrwick/ Porechop) was used for demultiplexing of barcoded reads and trimming of ONT 
adaptors. Genome assemblies were then constructed using SMARTdenovo (https:// github.com/ruanjue/smartdenovo) with parameters "-c 1 -k 14 -J 500 -e zmo". The assembly of strain 122 was polished using an Illumina MiSeq $2 \times 150$-bp paired-end sequencing dataset of the same strain and Pilon [148] (v1.23). Both assemblies were scaffolded further using ONT long-read information with SSPACE-LongRead [149] (v1-1) and, in the case of strain 122, gaps were closed using GapFiller [150] (v1-10) and the Illumina reads. The NBRC 1723 assembly was corrected by mapping the raw ONT reads using minimap2 [151] (2.17) and then using Racon [152] (v1.3.3). The final assemblies were generated after a second round of scaffolding using SSPACE-LongRead (v1-1), followed by polishing using Pilon (v1.23) for strain 122 or Nanopolish [153] (v0.11.0) for strain NBRC 1723.

\section{Gene annotation and analysis of meiotic gene content}

For the generation of a comprehensive $S d$. ludwigii gene annotation dataset, we used the reference genome sequence and combined the results from different tools and pipelines, followed by extensive manual curation. The methods and tools used are described in detail in the Additional file 10: Supplementary detailed methods. All S. cerevisiae genes that are known to be involved in meiosis and recombination were retrieved from the Saccharomyces Genome Database (SGD, https://www.yeastgenome.org) by searching for genes with relevant GO terms. These searches retrieved a total of 284 genes, which were then used as queries for the identification of homologs in the Sd. ludwigii gene annotation dataset using BLAST+ analyses. For all genes that remained undetected after similarity searches, we performed detailed gene synteny comparisons with annotated Saccharomycetaceae species that are included in the Yeast Gene Order Browser (YGOB) database (v7; http://ygob.ucd.ie). Further details are provided in the Additional file 10: Supplementary detailed methods.

\section{Meiotic segregation analysis}

Reads were mapped to the Sd. ludwigii reference genome (strain NBRC 1722), which was previously masked with RepeatMasker (v4.0.7, default parameters; http://www. repeatmasker.org), using bwa mem [154] (v0.7.17). Resulting bam files were sorted and indexed using SAMtools [155] (v1.9). Duplicate reads were marked and sample names were assigned using Picard (v2.18.14; https://broadinstitute.github.io/picard/). The GATK pipeline [156] (v3.7.0) was used to realign remaining reads. Variants were then called using GATK UnifiedGenotyper. Calling was performed simultaneously for all spores from the same tetrad or all lines from the same background.

For the single-nucleotide polymorphism (SNP) segregation analysis of the hybrid cross, SNPs called from the respective reads mapping were first filtered (bcftools view; v1.9, https://github.com/samtools/bcftools) in order to define a set of high-confidence discriminant markers. Only positions with a single alternate allele, supported by at least 10 reads across both parents and with $>90 \%$ of the reads covering either the reference or alternate allele, were selected. For each tetrad of the cross, SNPs located at the aforementioned marker positions were extracted, and the parental origin was assigned based on SNP correspondence between parents and spores at those positions. The result was formatted as a Seg file and used as input of the CrossOver pipeline (ReCombine suite) 
[157], using default parameters and modified chromosome sizes/coordinates to match the reference genome of $S d$. ludwigii. The reported events were individually validated by visual inspection using the Integrative Genomics Viewer (IGV). The same method was applied for the SNP segregation analysis of the South African cross, except that the minimum amount of reads supporting a marker position was lowered to 3 for each parent, as the coverage was reduced for one of the parents.

\section{Mutation accumulation analysis}

Filters were applied to the SNPs called from the mutation accumulation lines to identify SNPs that were present only in a single line, as we expected these events to be lineexclusive. First, only positions covered by more than 10 reads in each sample with a single alternate allele were kept. Then, filters based on the numbers of lines per background and the type of conversion event expected to occur in a given background (homozygous to homozygous, homozygous to heterozygous, heterozygous to homozygous) were applied. Heterozygous SNPs were only retained if their allele balance was between 0.4 and 0.6 . In the case of heterozygous-to-homozygous substitution, likely LOH events were filtered out to prevent false-positive SNP calls. The basesubstitutional mutation rate $\left(\mu_{\mathrm{bs}}\right)$ of each strain genealogy was calculated as the ratio of the total number of line-exclusive novel mutations divided by the size of the analyzed fraction of the genome (after masking), the total number of generations, and the number of respective lines. The $95 \%$ Poisson confidence intervals of mutation rates were computed as in Long et al. [158], and the mutation bias (corrected for the genomic GC content) as well as the theoretical equilibrium GC level (under mutation pressure alone) was calculated as in Krasovec et al. [159].

\section{Population analyses}

A total of 558,629 biallelic segregating sites were used to construct the neighbor-joining trees using the R packages ape and SNPrelate. The .gvcf matrices (of the whole genome or individual chromosomes) were converted into .gds files. Individual dissimilarities were estimated using the snpgdsDiss function, and the bionj algorithm was run on the distance matrices. Tree comparison was performed using the Visual TreeCmp package [160] (web application; v2.0.76), using 7 unrooted metrics (topological or weighted). For normalization of distances, the results of the topological metrics were compared to the average values of 1000 randomly generated tree pairs (uniform average method). The software PopLDdecay [161] (v3.41) was used for the calculation of the LD decay data, which were then smoothed (moving average method) and plotted using R.

\section{Other bioinformatic analyses}

Our methods and tools used for genomic comparisons, determination of structural variation, and identification of repetitive regions are provided in the Additional file 10: Supplementary detailed methods.

\section{Supplementary Information}

The online version contains supplementary material available at https://doi.org/10.1186/s13059-021-02521-w. 
Additional file 2: Figs. S1-S6.
Additional file 3: Table S2. Genome assemblies of yeasts and filamentous fungi.

Additional file 4: Table S3. Genes involved in meiosis/meiotic recombination and their presence/absence in Sd. ludwigii and other yeasts.

Additional file 5: Table S4. Population analyses: read mapping statistics.

Additional file 6: Table S5. Novel mutations in the mutation accumulation experiment.

Additional file 7: Table S6. $\mathrm{LOH}$ events in the mutation accumulation experiment.

Additional file 8: Table S7. List of plasmids.

Additional file 9: Table S8. List of PCR primers.

Additional file 10. Supplementary detailed methods.

Additional file 11. review history

\section{Acknowledgements}

We would like to acknowledge T. Yamazaki for his valuable feedback and useful advice on handling Sd. ludwigii, and K. T. Nishant for his input during revision of the manuscript. We are also grateful to A. Shinohara for the anti-Rad51 antibody, E. Mancera and L. Steinmetz for the anti-Rfa1 antibody, J. Trček for the Sd. ludwigii isolate BJK_5C, and A. Giacomini for the Sd. Iudwigii isolate PC99/R. Furthermore, we would like to thank Denise Zickler, Jean-Luc Souciet, and Bernard Dujon for their input, and Mani Arumugam, Wei Wu, Patrick Winker, Vladimir Benes, and Jean-Marc Aury for their help with genome sequencing during the early stages of this project. We also acknowledge support by the state of Baden-Württemberg through bwHPC for high-performance computing and SDS@hd for data storage (grant INST 35/1314-1 FUGG). Additional help was provided by the Flow Cytometry and the Deep Sequencing Core Facilities of the University of Heidelberg (both supported by CellNetworks).

\section{Review history}

The review history is available as Additional file 11.

\section{Peer review information}

Anahita Bishop was the primary editor of this article and managed its editorial process and peer review in collaboration with the rest of the editorial team.

\section{Authors' contributions}

Conceptualization: M.K.; Methodology: M.K., I.A.P., J.S. and A.F.; Investigation: I.A.P., F.A.P., H.M. and A.B.; Formal analysis: I.A.P., F.D., A.F. and N.D.; Supervision: M.K. and I.A.P.; Visualization: I.A.P., F.D. and A.F.; Writing-original draft: I.A.P.; Writing-review \& editing: I.A.P., M.K., J.S. and A.F.; Funding acquisition: M.K. and I.A.P. All authors read and approved the final manuscript.

\section{Funding}

This work was supported by a postdoctoral fellowship grant from the Cluster of Excellence CellNetworks (University of Heidelberg) to I.A.P. Open Access funding enabled and organized by Projekt DEAL.

\section{Availability of data and materials}

All sequencing datasets and genome assemblies generated and analyzed in this work are available at the NCBI databases, organized in BioProjects with accession numbers PRJNA28063 [162] (genomics and meiotic segregation analyses), PRJNA578491 [163] (mutation accumulation analysis), and PRJNA639224 [164] (population analyses). Correspondence and requests for materials should be addressed to M.K. (m.knop@zmbh.uni-heidelberg.de).

\section{Declarations}

Ethics approval and consent to participate

Not applicable.

Consent for publication

Not applicable.

\section{Competing interests}

The authors declare that they have no competing interests.

\section{Author details}

${ }^{1}$ Center for Molecular Biology of Heidelberg University (ZMBH), Heidelberg, Germany. ${ }^{2}$ Université de Strasbourg, CNRS, GMGM UMR 7156, Strasbourg, France. ${ }^{3}$ Current affiliation: Faculty of Agriculture, Kyushu University, Fukuoka, Japan. ${ }^{4}$ Umeå Plant Science Centre, Department of Forest Genetics and Plant Physiology, Swedish University of Agricultural Sciences, Umeå, Sweden. ${ }^{5}$ Institut Universitaire de France (IUF), Paris, France. ${ }^{6}$ German Cancer Research Center (DKFZ), DKFZ-ZMBH Alliance, Heidelberg, Germany. 
Received: 28 May 2021 Accepted: 20 October 2021

Published online: 03 November 2021

\section{References}

1. Otto SP. The evolutionary enigma of sex. Am Nat. 2009;174:S1-14.

2. Lenormand T, Engelstädter J, Johnston SE, Wijnker E, Haag CR. Evolutionary mysteries in meiosis. Philos Trans R SoC Lond Ser B Biol Sci. 2016;371:20160001.

3. Fisher RA. The genetical theory of natural selection. Oxford: Clarendon Press; 1930. p. xiv, 272.

4. Muller HJ. Some genetic aspects of sex. Am Nat. 1932;66:118-38.

5. Maynard SJ. The evolution of sex. Cambridge: Cambridge University Press; 1978.

6. Bell G. The masterpiece of nature: the evolution and genetics of sexuality: University of California Press; 1982.

7. Hartfield M, Keightley PD. Current hypotheses for the evolution of sex and recombination. Integr Zool. 2012;7:192-209.

8. Hunter N. Meiotic Recombination: The Essence of Heredity. Cold Spring Harb Perspect Biol. 2015:7:a016618.

9. Zickler D, Kleckner N. Recombination, pairing, and synapsis of homologs during meiosis. Cold Spring Harb Perspect Biol. 2015;7:a016626.

10. Keeney S, Giroux CN, Kleckner N. Meiosis-specific DNA double-strand breaks are catalyzed by Spo11, a member of a widely conserved protein family. Cell. 1997:88:375-84.

11. Yamazaki T, Ohara Y, Oshima Y. Rare occurrence of the tetratype tetrads in Saccharomycodes ludwigii. J Bacteriol. 1976; 125:461-6.

12. Yamazaki T, Oshima Y. Saccharomycodes ludwigii has seven chromosomes. Yeast. 1996;12:237-40.

13. Gray S, Cohen PE. Control of meiotic crossovers: from double-strand break formation to designation. Annu Rev Genet. 2016:50:175-210.

14. Phadnis N, Hyppa RW, Smith GR. New and old ways to control meiotic recombination. Trends Genet. 2011;27:411-21.

15. Wang S, Zickler D, Kleckner N, Zhang L. Meiotic crossover patterns: Obligatory crossover, interference and homeostasis in a single process. Cell Cycle. 2015;14:305-14.

16. Barnes TM, Kohara Y, Coulson A, Hekimi S. Meiotic recombination, noncoding DNA and genomic organization in Caenorhabditis elegans. Genetics. 1995;141:159-79.

17. Cromie G, Smith GR. Meiotic recombination in Schizosaccharomyces pombe: a paradigm for genetic and molecular analysis. Genome Dyn Stab. 2008;3:195.

18. Chakraborty P, Pankajam AV, Lin G, Dutta A, Krishnaprasad GN, Tekkedil MM, et al. Modulating crossover frequency and interference for obligate crossovers in Saccharomyces cerevisiae meiosis. G3. 2017;7:1511-24.

19. Neaves LE, Eales J, Whitlock R, Hollingsworth PM, Burke T, Pullin AS. The fitness consequences of inbreeding in natural populations and their implications for species conservation - a systematic map. Environ Evid. 2015:4:5.

20. Mogie M. Automixis: its distribution and status. Biol J Linn Soc. 1986;28:321-9.

21. Taxis C, Keller P, Kavagiou Z, Jensen L, Colombelli J, Bork P, et al. Spore number control and breeding in Saccharomyces cerevisiae: a key role for a self-organizing system. J Cell Biol. 2005;171:627-40.

22. Knop M. Evolution of the hemiascomycete yeasts: on life styles and the importance of inbreeding. BioEssays. 2006;28: 696-708.

23. Fischer G, Liti G, Llorente B. The budding yeast life cycle: more complex than anticipated? Yeast. 2021;38:5-11.

24. Zakharov IA. Increase in homozygosity as a result of intratetrad and intraoctad fertilization in fungi. Genet Mosc. 1968;4: 98-105.

25. Zakharov IA. Intratetrad mating and its genetic and evolutionary consequences. Russ J Genet. 2005:41:402-11.

26. Hood ME, Antonovics J. Mating within the meiotic tetrad and the maintenance of genomic heterozygosity. Genetics. 2004;166:1751-9.

27. Keller PJ, Knop M. Evolution of mutational robustness in the yeast genome: a link to essential genes and meiotic recombination hotspots. Malik HS, editor. PLoS Genet. 2009:5:e1000533.

28. Noda S. Achiasmate meiosis in the Fritillaria japonica group. Heredity. 1975;34:373-80.

29. Riddle NC, Elgin SCR. The dot chromosome of Drosophila: insights into chromatin states and their change over evolutionary time. Chromosom Res. 2006;14:405-16.

30. de la Fuente R, Parra MT, Viera A, Calvente A, Gómez R, Suja JÁ, et al. Meiotic pairing and segregation of achiasmate sex chromosomes in eutherian mammals: the role of SYCP3 protein. PLoS Genet. 2007;3.

31. Haag CR, Theodosiou L, Zahab R, Lenormand T. Low recombination rates in sexual species and sex-asex transitions. Philos Trans R Soc B Biol Sci. 2017;372:20160461.

32. Stapley J, Feulner PGD, Johnston SE, Santure AW, Smadja CM. Variation in recombination frequency and distribution across eukaryotes: patterns and processes. Philos Trans R Soc B Biol Sci. 2017;372:20160455.

33. Simmons RB, Ahearn DG. Ascospore Ornamentation in Saccharomycodes Ludwigii. Mycologia. 1985;77:660-2.

34. Winge O, Laustsen O. Saccharomycodes ludwigii Hansen, a balanced heterozygote. Comptes-Rendus Lab Carlsberg Ser Physiol. 1939;22:357-79.

35. Barnett JA. A history of research on yeasts 10: foundations of yeast genetics. Yeast. 2007;24:799-845.

36. Lindegren CC. Yeast genetics: Life cycles, cytology, hybridization, vitamin synthesis, and adaptive enzymes. Bacteriol Rev. 1945;9:111.

37. Ohara Y, Nonomura H, Yamazaki T. Dynamic aspect of yeast-flora during vinous fermentation: IX. Preferential isolation of wild yeasts (III). Bull Res Inst Ferment. 1964;11:1-12.

38. Štornik A, Skok B, Trček J. Comparison of cultivable acetic acid bacterial microbiota in organic and conventional apple cider vinegar. Food Technol Biotechnol. 2016;54:113-9.

39. Bovo B, Carlot M, Lombardi A, Lomolino G, Lante A, Giacomini A, et al. Exploring the use of Saccharomyces cerevisiae commercial strain and Saccharomycodes ludwigii natural isolate for grape marc fermentation to improve sensory properties of spirits. Food Microbiol. 2014;41:33-41.

40. Tavares MJ, Güldener U, Mendes-Ferreira A, Mira NP. Genome sequencing, annotation and exploration of the SO2tolerant non-conventional yeast Saccharomycodes ludwigii. BMC Genomics. 2021;22:131. 
41. Gabaldón T, Fairhead C. Genomes shed light on the secret life of Candida glabrata: not so asexual, not so commensal. Curr Genet. 2019;65:93-8.

42. Ouoba LII, Nielsen DS, Anyogu A, Kando C, Diawara B, Jespersen L, et al. Hanseniaspora jakobsenii sp. nov., a yeast isolated from Bandji, a traditional palm wine of Borassus akeassii. Int J Syst Evol Microbiol. 2015;65:3576-9.

43. Braun-Galleani S, Dias JA, Coughlan AY, Ryan AP, Byrne KP, Wolfe KH. Genomic diversity and meiotic recombination among isolates of the biotech yeast Komagataella phaffii (Pichia pastoris). Microb Cell Factories. 2019;18:211.

44. Gleeson MA, Sudbery PE. Genetic analysis in the methylotrophic yeast Hansenula polymorpha. Yeast. 1988;4:293-303.

45. Brion C, Legrand S, Peter J, Caradec C, Pflieger D, Hou J, et al. Variation of the meiotic recombination landscape and properties over a broad evolutionary distance in yeasts. Fung JC, editor. PLoS Genet. 2017;13:e1006917.

46. Zonneveld BJM, Steensma HY. Mating, sporulation and tetrad analysis in Kluyveromyces lactis. In: Wolf K, Breunig K, Barth G, editors. Non-Conv Yeasts Genet Biochem Biotechnol Pract Protoc. Berlin, Heidelberg: Springer; 2003. p. 151-4.

47. Lam I, Keeney S. Mechanism and regulation of meiotic recombination initiation. Cold Spring Harb Perspect Biol. 2014;7: a016634.

48. Engebrecht J, Voelkel-Meiman K, Roeder GS. Meiosis-specific RNA splicing in yeast. Cell. 1991;66:1257-68.

49. Engebrecht J, Roeder GS. Yeast Mer1 Mutants display reduced levels of meiotic recombination. Genetics. 1989;121:237-47.

50. Deshpande RA, Wilson TE. Modes of interaction among yeast Nej1, Lif1 and Dn/4 proteins and comparison to human XLF, XRCC4 and Lig4. DNA Repair. 2007;6:1507-16.

51. Stegmeier F, Visintin R, Amon A. Separase, polo kinase, the kinetochore protein SIk19, and Spo12 function in a network that controls Cdc14 localization during early anaphase. Cell. 2002;108:207-20.

52. Cuperus G, Shore D. Restoration of silencing in Saccharomyces cerevisiae by tethering of a novel Sir2-interacting protein, Esc8. Genetics. 2002;162:633-45.

53. Steenwyk JL, Opulente DA, Kominek J, Shen X-X, Zhou X, Labella AL, et al. Extensive loss of cell-cycle and DNA repair genes in an ancient lineage of bipolar budding yeasts. PLoS Biol. 2019;17:e3000255.

54. Klapholz S, Waddell CS, Esposito RE. The role of the Spo11 gene in meiotic recombination in yeast. Genetics. 1985;110: $187-216$.

55. Rockmill B, Lefrançois $P$, Voelkel-Meiman $\mathrm{K}$, Oke A, Roeder GS, Fung JC. High throughput sequencing reveals alterations in the recombination signatures with diminishing Spo11 activity. PLoS Genet. 2013;9:e1003932.

56. Cannavo E, Cejka P. Sae2 promotes dsDNA endonuclease activity within Mre11-Rad50-Xrs2 to resect DNA breaks. Nature. 2014;514:122-5.

57. Brown MS, Grubb J, Zhang A, Rust MJ, Bishop DK. Small Rad51 and Dmc1 complexes often co-occupy both ends of a meiotic DNA double strand break. PLoS Genet. 2015;11:e1005653.

58. Bishop DK. RecA homologs Dmc1 and Rad51 interact to form multiple nuclear complexes prior to meiotic chromosome synapsis. Cell. 1994;79:1081-92.

59. Johnson R, Borde V, Neale MJ, Bishop-Bailey A, North M, Harris S, et al. Excess single-stranded DNA inhibits meiotic double-strand break repair. PLoS Genet. 2007;3:e223.

60. Spies M, Fishel R. Mismatch repair during homologous and homeologous recombination. Cold Spring Harb Perspect Biol. 2015;7:a022657.

61. Cooper TJ, Crawford MR, Hunt LJ, Marsolier-Kergoat M-C, Llorente B, Neale MJ. Mismatch repair impedes meiotic crossover interference. BioRxiv. 2018;480418.

62. Mancera E, Bourgon R, Brozzi A, Huber W, Steinmetz LM. High-resolution mapping of meiotic crossovers and noncrossovers in yeast. Nature. 2008;454:479-85.

63. Martini E, Borde V, Legendre M, Audic S, Regnault B, Soubigou G, et al. Genome-wide analysis of heteroduplex DNA in mismatch repair-deficient yeast cells reveals novel properties of meiotic recombination pathways. PLoS Genet. 2011;7: e1002305.

64. Klein F, Laroche T, Cardenas ME, Hofmann JF, Schweizer D, Gasser SM. Localization of RAP1 and topoisomerase II in nuclei and meiotic chromosomes of yeast. J Cell Biol. 1992;117:935-48.

65. Shore D. RAP1: a protean regulator in yeast. Trends Genet. 1994;10:408-12.

66. Schacherer J, Shapiro JA, Ruderfer DM, Kruglyak L. Comprehensive polymorphism survey elucidates population structure of Saccharomyces cerevisiae. Nature. 2009;458:342-5.

67. Friedrich A, Jung P, Reisser C, Fischer G, Schacherer J. Population genomics reveals chromosome-scale heterogeneous evolution in a protoploid yeast. Mol Biol Evol. 2015;32:184-92.

68. Lynch M, Sung W, Morris K, Coffey N, Landry CR, Dopman EB, et al. A genome-wide view of the spectrum of spontaneous mutations in yeast. Proc Natl Acad Sci. 2008;105:9272-7.

69. Nguyen DT, Wu B, Long H, Zhang N, Patterson C, Simpson S, et al. Variable spontaneous mutation and loss of heterozygosity among heterozygous genomes in yeast. Mol Biol Evol. 2020;37:3118-30.

70. Katju V, Bergthorsson U. Old trade, new tricks: insights into the spontaneous mutation process from the partnering of classical mutation accumulation experiments with high-throughput genomic approaches. Genome Biol Evol. 2019;11: $136-65$.

71. Sharp NP, Sandell L, James CG, Otto SP. The genome-wide rate and spectrum of spontaneous mutations differ between haploid and diploid yeast. Proc Natl Acad Sci. 2018;115:E5046-55.

72. Tattini L, Tellini N, Mozzachiodi S, D'Angiolo M, Loeillet S, Nicolas A, et al. Accurate tracking of the mutational landscape of diploid hybrid genomes. Mol Biol Evol. 2019;36:2861-77.

73. James TY, Michelotti LA, Glasco AD, Clemons RA, Powers RA, James ES, et al. Adaptation by loss of heterozygosity in saccharomyces cerevisiae clones under divergent selection. Genetics. 2019;213:665-83.

74. Zhang K, Zheng D-Q, Sui Y, Qi L, Petes TD. Genome-wide analysis of genomic alterations induced by oxidative DNA damage in yeast. Nucleic Acids Res. 2019;47:3521-35.

75. St Charles J, Petes TD. High-resolution mapping of spontaneous mitotic recombination hotspots on the $1.1 \mathrm{Mb}$ arm of yeast chromosome IV. PLoS Genet. 2013;9:e1003434.

76. Mehta A, Haber JE. Sources of DNA double-strand breaks and models of recombinational DNA repair. Cold Spring Harb Perspect Biol. 2014;6:a016428. 
77. Goldfarb T, Lichten M. Frequent and efficient use of the sister chromatid for DNA double-strand break repair during budding yeast meiosis. PLoS Biol. 2010;8:e1000520.

78. Youds $J \mathrm{~L}$, Boulton $\mathrm{SJ}$. The choice in meiosis - defining the factors that influence crossover or non-crossover formation. J Cell Sci. 2011;124:501-13.

79. Chen J-M, Cooper DN, Chuzhanova N, Férec C, Patrinos GP. Gene conversion: mechanisms, evolution and human disease. Nat Rev Genet. 2007;8:762-75.

80. Joyce EF, Paul A, Chen KE, Tanneti N, McKim KS. Multiple barriers to nonhomologous DNA end joining during meiosis in Drosophila. Genetics. 2012;191:739-46.

81. Yun H, Kim K. Ku complex suppresses recombination in the absence of MRX activity during budding yeast meiosis. BMB Rep. 2019;52:607-12.

82. Johnson $\sqcup$, Antonovics J, Hood ME. The evolution of intratetrad mating rates. Evolution. 2005;59:2525-32.

83. Winge Ö. The segregation in the ascus ofSaccharomycodes Ludwigii. Antonie Van Leeuwenhoek. 1947;12:129-32.

84. Kreger-van Rij NJ. A new feature of the ascospores of Saccharomycodes ludwigii Hansen. Can J Microbiol. 1969;15:823-5.

85. Deutschbauer AM, Williams RM, Chu AM, Davis RW. Parallel phenotypic analysis of sporulation and postgermination growth in Saccharomyces cerevisiae. Proc Natl Acad Sci. 2002;99:15530-5.

86. Tsubouchi T, Zhao H, Roeder GS. The meiosis-specific zip4 protein regulates crossover distribution by promoting synaptonemal complex formation together with zip2. Dev Cell. 2006;10:809-19.

87. Chelysheva L, Gendrot G, Vezon D, Doutriaux M-P, Mercier R, Grelon M. Zip4/Spo22 is required for class I CO formation but not for synapsis completion in Arabidopsis thaliana. PLoS Genet. 2007;3:e83.

88. Adelman CA, Petrini JHJ. ZIP4H (TEX11) Deficiency in the mouse impairs meiotic double strand break repair and the regulation of crossing over. PLoS Genet. 2008:4:e1000042.

89. Shen Y, Tang D, Wang K, Wang M, Huang J, Luo W, et al. ZIP4 in homologous chromosome synapsis and crossover formation in rice meiosis. J Cell Sci. 2012;125:2581-91.

90. Novak JE, Ross-Macdonald PB, Roeder GS. The budding yeast Msh4 protein functions in chromosome synapsis and the regulation of crossover distribution. Genetics. 2001;158:1013-25.

91. de los Santos T, Hunter N, Lee C, Larkin B, Loidl J, Hollingsworth NM. The Mus81/Mms4 endonuclease acts independently of double-Holliday junction resolution to promote a distinct subset of crossovers during meiosis in budding yeast. Genetics. 2003;164:81-94.

92. Kurtzman C, Fell JW. The yeasts - a taxonomic study. 4th Edition. Elsevier Science; 1998.

93. Klapholz S, Esposito RE. Isolation of SPO12-1 and SPO13-1 from a natural variant of yeast that undergoes a single meiotic division. Genetics. 1980;96:567-88.

94. Munz P, Wolf K, Kohli J, Leupold U. Genetics overview. Mol Biol Fission Yeast. San Diego: Academic Press. 1989:1-30

95. Marsolier-Kergoat M-C, Yeramian E. GC Content and recombination: reassessing the causal effects for the Saccharomyces cerevisiae genome. Genetics. 2009;183:31-8.

96. Lynch M. Evolution of the mutation rate. Trends Genet. 2010;26:345-52.

97. Hildebrand F, Meyer A, Eyre-Walker A. Evidence of selection upon genomic GC-content in bacteria. PLoS Genet. 2010;6: e1001107.

98. Liu H, Zhang J. Yeast spontaneous mutation rate and spectrum vary with environment. Curr Biol. 2019;29:1584-1591.e3.

99. Lancaster SM, Payen C, Heil CS, Dunham MJ. Fitness benefits of loss of heterozygosity in Saccharomyces hybrids. Genome Res. 2019;29:1685-92.

100. Peter J, De Chiara M, Friedrich A, Yue J-X, Pflieger D, Bergström A, et al. Genome evolution across 1011 Saccharomyces cerevisiae isolates. Nature. 2018;556:339-44.

101. Page SL, Hawley RS. The genetics and molecular biology of the synaptonemal complex. Annu Rev Cell Dev Biol. 2004; 20:525-58.

102. Agarwal S, Roeder GS. Zip3 provides a link between recombination enzymes and synaptonemal complex proteins. Cell. 2000;102:245-55.

103. Niu H, Wan L, Baumgartner B, Schaefer D, Loidl J, Hollingsworth NM. Partner choice during meiosis is regulated by Hop1-promoted dimerization of Mek1. Mol Biol Cell. 2005;16:5804-18.

104. Chua PR, Roeder GS. Tam1, a telomere-associated meiotic protein, functions in chromosome synapsis and crossover interference. Genes Dev. 1997;11:1786-800.

105. Shinohara M, Oh SD, Hunter N, Shinohara A. Crossover assurance and crossover interference are distinctly regulated by the ZMM proteins during yeast meiosis. Nat Genet. 2008;40:299-309.

106. Sikorski RS, Hieter P. A system of shuttle vectors and yeast host strains designed for efficient manipulation of DNA in Saccharomyces cerevisiae. Genetics. 1989;122:19-27.

107. Wach A, Brachat A, Pöhlmann R, Philippsen P. New heterologous modules for classical or PCR-based gene disruptions in Saccharomyces cerevisiae. Yeast. 1994;10:1793-808.

108. Janke C, Magiera MM, Rathfelder N, Taxis C, Reber S, Maekawa H, et al. A versatile toolbox for PCR-based tagging of yeast genes: new fluorescent proteins, more markers and promoter substitution cassettes. Yeast. 2004;21:947-62.

109. Knop M, Siegers K, Pereira G, Zachariae W, Winsor B, Nasmyth K, et al. Epitope tagging of yeast genes using a PCRbased strategy: more tags and improved practical routines. Yeast. 1999;15:963-72.

110. Neiman AM. Prospore membrane formation defines a developmentally regulated branch of the secretory pathway in yeast. J Cell Biol. 1998;140:29-37.

111. Raghavan V, Bui DT, Al-Sweel N, Friedrich A, Schacherer J, Aquadro CF, et al. Incompatibilities in mismatch repair genes MLH1-PMS1 contribute to a wide range of mutation rates in human isolates of baker's yeast. Genetics. 2018;210:1253-66

112. Schneider CA, Rasband WS, Eliceiri KW. NIH Image to ImageJ: 25 years of image analysis. Nat Methods. 2012;9:671-5.

113. Proux-Wéra E, Armisén D, Byrne KP, Wolfe KH. A pipeline for automated annotation of yeast genome sequences by a conserved-synteny approach. BMC Bioinformatics. 2012;13:237.

114. Byrne KP, Wolfe KH. The Yeast Gene Order Browser: combining curated homology and syntenic context reveals gene fate in polyploid species. Genome Res. 2005;15:1456-61.

115. Stanke M, Morgenstern B. AUGUSTUS: a web server for gene prediction in eukaryotes that allows user-defined constraints. Nucleic Acids Res. 2005;33:W465-7. 
116. Cantarel BL, Korf I, Robb SMC, Parra G, Ross E, Moore B, et al. MAKER: An easy-to-use annotation pipeline designed for emerging model organism genomes. Genome Res. 2008;18:188-96.

117. Lomsadze A, Ter-Hovhannisyan V, Chernoff $Y$, Borodovsky M. Gene identification in novel eukaryotic genomes by selftraining algorithm. Nucleic Acids Res. 2005;33:6494-506.

118. Korf I. Gene finding in novel genomes. BMC Bioinformatics. 2004;5:59

119. Thorvaldsdóttir H, Robinson JT, Mesirov JP. Integrative Genomics Viewer (IGV): high-performance genomics data visualization and exploration. Brief Bioinform. 2013;14:178-92.

120. Camacho C, Coulouris G, Avagyan V, Ma N, Papadopoulos J, Bealer K, et al. BLAST+: architecture and applications. BMC Bioinformatics. 2009;10:421.

121. Quinlan AR, Hall IM. BEDTools: a flexible suite of utilities for comparing genomic features. Bioinformatics. 2010;26:841-2.

122. Lowe TM, Chan PP. tRNAscan-SE On-line: integrating search and context for analysis of transfer RNA genes. Nucleic Acids Res. 2016;44:W54-7.

123. Lagesen $\mathrm{K}$, Hallin $\mathrm{P}$, Rødland $\mathrm{E}$, Stærfeldt H-H, Rognes T, Ussery D. RNAmmer: consistent and rapid annotation of ribosomal RNA genes. Nucleic Acids Res. 2007;35:3100-8.

124. Kalvari I, Argasinska J, Quinones-Olvera N, Nawrocki EP, Rivas E, Eddy SR, et al. Rfam 13.0: shifting to a genome-centric resource for non-coding RNA families. Nucleic Acids Res. 2018;46:D335-42.

125. Waterhouse RM, Seppey M, Simão FA, Manni M, loannidis P, Klioutchnikov G, et al. BUSCO applications from quality assessments to gene prediction and phylogenomics. Mol Biol Evol. 2018;35:543-8.

126. Götz S, García-Gómez JM, Terol J, Williams TD, Nagaraj SH, Nueda MJ, et al. High-throughput functional annotation and data mining with the Blast2GO suite. Nucleic Acids Res. 2008;36:3420-35.

127. Jones $\mathrm{P}$, Binns D, Chang H-Y, Fraser M, Li W, McAnulla C, et al. InterProScan 5: genome-scale protein function classification. Bioinformatics. 2014;30:1236-40

128. Huerta-Cepas J, Szklarczyk D, Forslund K, Cook H, Heller D, Walter MC, et al. eggNOG 4.5: a hierarchical orthology framework with improved functional annotations for eukaryotic, prokaryotic and viral sequences. Nucleic Acids Res. 2016:44:D286-93.

129. Gordon JL, Byrne KP, Wolfe KH. Mechanisms of chromosome number evolution in yeast. PLoS Genet. 2011:7:e1002190

130. Douglass AP, Byrne KP, Wolfe KH. The Methylotroph Gene Order Browser (MGOB) reveals conserved synteny and ancestral centromere locations in the yeast family Pichiaceae. FEMS Yeast Res. 2019:19:foz058.

131. Shen X-X, Opulente DA, Kominek J, Zhou X, Steenwyk JL, Buh KV, et al. Tempo and mode of genome evolution in the budding yeast subphylum. Cell. 2018;175:1533-1545.e20.

132. Cherry JM, Hong EL, Amundsen C, Balakrishnan R, Binkley G, Chan ET, et al. Saccharomyces Genome Database: the genomics resource of budding yeast. Nucleic Acids Res. 2012;40:D700-5.

133. El-Gebali S, Mistry J, Bateman A, Eddy SR, Luciani A, Potter SC, et al. The Pfam protein families database in 2019. Nucleic Acids Res. 2019;47:D427-32.

134. Mészáros B, Erdos G, Dosztányi Z. IUPred2A: context-dependent prediction of protein disorder as a function of redox state and protein binding. Nucleic Acids Res. 2018;46:W329-37.

135. Marçais G, Delcher AL, Phillippy AM, Coston R, Salzberg SL, Zimin A. MUMmer4: A fast and versatile genome alignment system. PLoS Comput Biol. 2018;14:e1005944.

136. Gu Z, Gu L, Eils R, Schlesner M, Brors B. circlize implements and enhances circular visualization in R. Bioinformatics. 2014; 30:2811-2

137. Sedlazeck FJ, Rescheneder P, Smolka M, Fang H, Nattestad M, von Haeseler A, et al. Accurate detection of complex structural variations using single-molecule sequencing. Nat Methods. 2018;15:461-8.

138. Li H. Aligning sequence reads, clone sequences and assembly contigs with BWA-MEM. ArXiv Genomics Q-BioGN. 2013; 1303:3997v2.

139. Hubley R, Finn RD, Clements J, Eddy SR, Jones TA, Bao W, et al. The Dfam database of repetitive DNA families. Nucleic Acids Res. 2016:44:D81-9.

140. Bao W, Kojima KK, Kohany O. Repbase Update, a database of repetitive elements in eukaryotic genomes. Mob DNA. 2015:6:11.

141. Benson G. Tandem repeats finder: a program to analyze DNA sequences. Nucleic Acids Res. 1999;27:573-80.

142. R Core Team. R: A language and environment for statistical computing [Internet]. Vienna: R Foundation for Statistical Computing; 2020. [cited 2020 Sep 11]. Available from: URL https://www.R-project.org/

143. Huber W, Carey VJ, Gentleman R, Anders S, Carlson M, Carvalho BS, et al. Orchestrating high-throughput genomic analysis with Bioconductor. Nat Methods. 2015;12:115-21.

144. Loidl J, Klein F, Scherthan H. Homologous pairing is reduced but not abolished in asynaptic mutants of yeast. J Cell Biol. 1994;125:1191-200

145. Shinohara A, Ogawa H, Ogawa T. Rad51 protein involved in repair and recombination in S. cerevisiae is a RecA-like protein. Cell. 1992;69:457-70.

146. Myers EW, Sutton GG, Delcher AL, Dew IM, Fasulo DP, Flanigan MJ, et al. A whole-genome assembly of Drosophila. Science. 2000;287:2196-204

147. Chin C-S, Alexander DH, Marks P, Klammer AA, Drake J, Heiner C, et al. Nonhybrid, finished microbial genome assemblies from long-read SMRT sequencing data. Nat Methods. 2013;10:563-9.

148. Walker BJ, Abeel T, Shea T, Priest M, Abouelliel A, Sakthikumar S, et al. Pilon: an integrated tool for comprehensive microbial variant detection and genome assembly improvement. PLoS One. 2014;9:e112963.

149. Boetzer M, Pirovano W. SSPACE-LongRead: scaffolding bacterial draft genomes using long read sequence information. BMC Bioinformatics. 2014;15:211

150. Boetzer M, Pirovano W. Toward almost closed genomes with GapFiller. Genome Biol. 2012;13:R56.

151. Li H. Minimap2: pairwise alignment for nucleotide sequences. Bioinformatics. 2018;34:3094-100.

152. Vaser R, Sović I, Nagarajan N, Šikić M. Fast and accurate de novo genome assembly from long uncorrected reads. Genome Res. 2017;27:737-46.

153. Loman NJ, Quick J, Simpson JT. A complete bacterial genome assembled de novo using only nanopore sequencing data. Nat Methods. 2015;12:733-5. 
154. Li H, Durbin R. Fast and accurate short read alignment with Burrows-Wheeler transform. Bioinformatics. 2009;25:1754-60

155. Li H, Handsaker B, Wysoker A, Fennell T, Ruan J, Homer N, et al. The Sequence Alignment/Map format and SAMtools. Bioinformatics. 2009;25:2078-9.

156. McKenna A, Hanna M, Banks E, Sivachenko A, Cibulskis K, Kernytsky A, et al. The Genome Analysis Toolkit: a MapReduce framework for analyzing next-generation DNA sequencing data. Genome Res. 2010;20:1297-303.

157. Anderson CM, Chen SY, Dimon MT, Oke A, DeRisi JL, Fung JC. ReCombine: a suite of programs for detection and analysis of meiotic recombination in whole-genome datasets. PLoS One. 2011;6:e25509.

158. Long $\mathrm{H}$, Behringer MG, Williams $\mathrm{E}$, Te R, Lynch M. Similar mutation rates but highly diverse mutation spectra in ascomycete and basidiomycete yeasts. Genome Biol Evol. 2016;8:3815-21.

159. Krasovec M, Sanchez-Brosseau S, Piganeau G. First estimation of the spontaneous mutation rate in diatoms. Genome Biol Evol. 2019;11:1829-37.

160. Goluch T, Bogdanowicz D, Giaro K. Visual TreeCmp: comprehensive comparison of phylogenetic trees on the web. Methods Ecol Evol. 2020;11:494-9.

161. Zhang C, Dong S-S, Xu J-Y, He W-M, Yang T-L. PopLDdecay: a fast and effective tool for linkage disequilibrium decay analysis based on variant call format files. Bioinformatics. 2019;35:1786-8.

162. Papaioannou IA, Knop M. Genomics and meiotic segregation study of Saccharomycodes ludwigii. NCBI BioProject dataset PRJNA28063 [Internet]. 2020; Available from: https://www.ncbi.nlm.nih.gov/bioproject/PRJNA28063.

163. Papaioannou IA, Knop M. Saccharomycodes ludwigii: mutation accumulation study. NCBI BioProject dataset PRJNA578491 [Internet]. 2020; Available from: https://www.ncbi.nlm.nih.gov/bioproject/PRJNA578491.

164. Papaioannou IA, Knop M. Saccharomycodes ludwigii: comparative genomics of wild-type isolates. NCBI BioProject dataset PRJNA639224 [Internet]. 2021; Available from: https://www.ncbi.nIm.nih.gov/bioproject/PRJNA639224.

\section{Publisher's Note}

Springer Nature remains neutral with regard to jurisdictional claims in published maps and institutional affiliations.

Ready to submit your research? Choose BMC and benefit from:

- fast, convenient online submission

- thorough peer review by experienced researchers in your field

- rapid publication on acceptance

- support for research data, including large and complex data types

- gold Open Access which fosters wider collaboration and increased citations

- maximum visibility for your research: over $100 \mathrm{M}$ website views per year

At BMC, research is always in progress.

Learn more biomedcentral.com/submissions 